\title{
THE APPROXIMATION THEOREM AND THE $K$-THEORY OF GENERALIZED FREE PRODUCTS
}

\author{
ROLAND SCHWÄNZL AND ROSS E. STAFFELDT
}

\begin{abstract}
We use methods of abstract algebraic $K$-theory as developed by Friedhelm Waldhausen to give a new derivation of the decomposition theorem for the algebraic $K$-theory of a generalized free product ring. The result takes the form of a fibration sequence which relates the algebraic $K$-theory of such a ring with the algebraic $K$-theory of its factors, plus a Nil-term.
\end{abstract}

\section{INTRODUCTION}

One of the main results of [8] is a decomposition of the $K$-theory of a ring which is a generalized free product in terms of the $K$-theories of the constituent rings, and one other object, which is generally called the Nil-term. We recall this in the fibration square described below in Theorem 4. Underlying the derivation of the square given in [8] is the construction of a suitable fibration. Besides a combinatorial analysis of a generalized free product, the construction depends on an ad hoc development of some parts of abstract algebraic $K$-theory, later systematized and generalized in [9]. It is the purpose of this paper to show how to derive the main theorems of [8] as applications of results and methods of [9]. Using different methods, Pierre Vogel [7] has also reconsidered results of [8].

We first develop language so that essentially the same fibration used in [8] may be derived from a general fibration theorem (Theorem 1.6.4 of [9, page 354]) developed as part of the overall approach to abstract algebraic $K$-theory described in [9]. This is Proposition 2.1 below. Our main contribution is the description of how the approximation theorem (Theorem 1.6.7 of [9, page 354]) may then be used to interpret terms in the fibration. These results are Theorems 2 and 3 stated at the end of the section. We are able to replace the technical maneuvering required in the original proofs with arguments that follow a standard pattern and are more conceptual. The paper also provides an introduction to a few of the ideas we will use in [5], where we generalize the situation to the case of simplicial rings. One of the goals of [5] is to set up a framework which will also allow us to handle decomposition problems in the $K$-theory of $A_{\infty}$-rings (informally, rings-up-to-homotopy), including partial analysis of the Nil terms. To these ends, several parts of the program in the present paper have

Received by the editors July 11, 1994.

1991 Mathematics Subject Classification. Primary 19D10; Secondary 19D35.

Key words and phrases. Abstract algebraic $K$-theory, generalized free products, approximation theorem.

Research of the second author supported by SFB 343 and an NMSU minigrant. 
to be treated differently in [5], with the result that the specialization of those proofs to the case of ordinary rings does not immediately make the underlying principles clear.

We will concern ourselves only with the case of a generalized free product of rings, but it will be clear that the methods also reprove the results for Laurent extensions and for polynomial extensions which are found in Sections 12 and 13 of [8], respectively.

We start with a diagram

$$
A \stackrel{\alpha}{\longleftarrow} C \stackrel{\beta}{\longrightarrow} B
$$

of discrete rings satisfying the freeness and purity conditions of [8]. That is, we require for purity that $\alpha: C \longrightarrow A$ be injective and that there be a splitting $A=\alpha(C) \oplus A^{\prime}$ of $C$-bimodules. Though the splitting is not part of the data and only its existence is required, it is convenient to refer to a fixed complement $A^{\prime}$ of $\alpha(C)$ in $A$. The freeness condition we will impose is that $A^{\prime}$ shall be free as a left $C$-module. We impose the same conditions on $\beta: C \longrightarrow B$.

Let

$$
R=A *_{C} B
$$

be the free product as displayed in [8]. As a $C$-bimodule, then

$$
\begin{aligned}
R= & C \oplus A^{\prime} \oplus B^{\prime} \oplus A^{\prime} \otimes_{C} B^{\prime} \oplus B^{\prime} \otimes_{C} A^{\prime} \oplus A^{\prime} \\
& \otimes_{C} B^{\prime} \otimes_{C} A^{\prime} \oplus B^{\prime} \otimes_{C} A^{\prime} \otimes_{C} B^{\prime} \oplus \cdots,
\end{aligned}
$$

and the problem is to describe the $K$-theory of $R$ in terms of the $K$-theories of $A, B$, and $C$.

In this paper $K$-theory is defined in terms of the $S_{\bullet}$ construction of [9], which from a category with cofibrations and weak equivalences produces its $K$-theory space. We consider certain categories of simplicial modules over the rings $A, B, C$, and $R$. These categories, whose definitions we recall in Section 2, will be denoted by $\mathscr{M}_{f}(A), \mathscr{M}_{f}(B), \mathscr{M}_{f}(C)$, and $\mathscr{M}_{f}(R)$, respectively. Each of these categories then supports a notion of cofibration, which we also recall below, and a weak equivalence in each case will be a homotopy equivalence, or, briefly, an $h$-map. Then the $K$-theory of $\mathscr{M}_{f}(A)$ with respect to these notions of cofibration and weak equivalence (generically indicated by the presence of an $h$ somewhere in the symbol) is defined in [9] as

$$
K\left(\mathscr{M}_{f}(A) ; h\right)=\Omega\left|h S_{\bullet} \mathscr{M}_{f}(A)\right| .
$$

According to Theorem 2.3.2 in [9, page 39.4] the relation with Quillen's plus construction definition of $K$-theory is

$$
\Omega\left|h S_{\bullet} \mathscr{M}_{f}(A)\right| \simeq K_{0}^{\prime}(A) \times B G L(A)^{+},
$$

where $K_{0}^{\prime}(A)$ is the subgroup of the usual Grothendieck group of isomorphism classes of projective modules $K_{0}(A)$ generated by the free modules. Therefore, we will abbreviate

$$
K\left(\mathscr{M}_{f}(A) ; h\right)=K(A)
$$

and use similar notations for the other rings $B, C$, and $R$.

We next describe the auxiliary categories which are essential to the proofs. First of all, we have the category of Mayer-Vietoris presentations $\mathscr{M} V$, in 
which an object is a sextuple $\left(M, M_{A}, M_{B}, M_{C}, l, \kappa\right)$, where $M$ is an object of $\mathscr{M}_{f}(R)$, and $M_{A}, M_{B}$, and $M_{C}$ are similarly objects of $\mathscr{M}_{f}(A), \mathscr{M}_{f}(B)$, and $\mathscr{M}_{f}(C)$, respectively. Also, $l$ and $\kappa$ are maps of $R$-modules such that

$$
0 \longrightarrow M \stackrel{l}{\longrightarrow} M_{A} \otimes_{A} R \oplus M_{B} \otimes_{B} R \stackrel{\kappa}{\longrightarrow} M_{C} \otimes_{C} R \longrightarrow 0
$$

is a fibration sequence of simplicial $R$-modules and

$$
\kappa\left(M_{A}\right) \subset M_{C} \otimes_{C} A \text { and } \kappa\left(M_{B}\right) \subset M_{C} \otimes_{C} B .
$$

These are the same conditions imposed in [8, page 142], but now taken degreewise. We will also find it useful to write $\kappa$ as $\kappa=\kappa_{\alpha}-\kappa_{\beta}$, where $\kappa_{\alpha}\left(M_{B}\right)=0$ and $\kappa_{\beta}\left(M_{A}\right)=0$. A map of Mayer-Vietoris presentations is a quadruple of maps $\left(f, f_{A}, f_{B}, f_{C}\right)$ in the respective module categories, such that the resulting ladder diagram commutes. By means of the forgetful functors to the respective module categories $\mathscr{M}_{f}(A), \mathscr{M}_{f}(B), \mathscr{M}_{f}(C)$, and $\mathscr{M}_{f}(R)$, one may define cofibrations in $\mathscr{M} V$. We discuss this in more detail in Section 2.

We will be working with two notions of weak equivalence in the category $\mathscr{M} V$. A map of Mayer-Vietoris presentations $\left(f, f_{A}, f_{B}, f_{C}\right)$ is a coarse weak equivalence if the map $f$ is a homotopy equivalence. The subcategory of these maps will be denoted by $w \mathscr{M} V$, and such a map will be called a $w$-map for short. A map of Mayer-Vietoris presentations is a fine weak equivalence, or a $v$-map, for short, if the maps $f_{A}, f_{B}$, and $f_{C}$ are homotopy equivalences. We denote the subcategory of $\mathscr{M} V$ where the arrows are the $v$-equivalences by $v \mathscr{M} V$. Notice that it follows from the five lemma that a $v$-map is also a $w$-map, so that $v \mathscr{M} V \subset w \mathscr{M} V$.

In Section 2, Proposition 2.1, we observe that Theorem 1.6.4 of [9, page 350] provides the following fibration-up-to-homotopy:

$$
v S_{\bullet} \mathscr{M} V^{w} \longrightarrow v S_{\bullet} \mathscr{M} V \longrightarrow w S_{\bullet} \mathscr{M} V,
$$

where $\mathscr{M} V^{w}$ denotes the subcategory of $\mathscr{M} V$ consisting of Mayer-Vietoris presentations in which the $R$-module is contractible. After taking loop spaces of the realizations, from left to right these terms are by definition the $K$-theory of the category of Mayer-Vietoris presentations of contractible $R$-modules, the $K$-theory of the category of Mayer-Vietoris presentations with respect to the $v$-equivalences (the fine equivalences) and the $K$-theory of the category of Mayer-Vietoris presentations with respect to the $w$-equivalences (the coarse equivalences).

In Section 2 we prove this first interpretative theorem.

Theorem 1. The forgetful functors from $\mathscr{M} V$ to the module categories $\mathscr{M}_{f}(A)$, $\mathscr{M}_{f}(B)$, and $\mathscr{M}_{f}(C)$ induce a homotopy equivalence

$$
u_{*}: v S_{\bullet} \mathscr{M} V \longrightarrow h S_{\bullet} \mathscr{M}_{f}(A) \times h S_{\bullet} \mathscr{M}_{f}(B) \times h S_{\bullet} \mathscr{M}_{f}(C) .
$$

In Section 4 we make our first use of the approximation theorem and prove this result.

Theorem 2. The forgetful functor

$$
u_{R}: \mathscr{M} V \longrightarrow \mathscr{M}_{f}(R)
$$


induces a homotopy equivalence

$$
w S_{\bullet} \mathscr{M} V \longrightarrow h S_{\bullet} \mathscr{M}_{f}(R) .
$$

Finally, we define $\mathscr{S}$, the category of split modules. This subcategory of $\mathscr{M} V$ consists of the Mayer-Vietoris presentations

$$
0 \longrightarrow 0 \stackrel{\iota}{\longrightarrow} M_{A} \otimes_{A} R \oplus M_{B} \otimes_{B} R \stackrel{\kappa}{\longrightarrow} M_{C} \otimes_{C} R \longrightarrow 0 .
$$

Alternatively, one characterizes objects of this category as Mayer-Vietoris presentations in which the map $\kappa$ is an isomorphism. This subcategory of $\mathscr{M} V$ inherits all the structures which are on $\mathscr{M} V$, but, of course, only the $v$ equivalences are relevant. Obviously it is a subcategory of $\mathscr{M} V^{w}$.

In Section 5 we prove our second main result.

Theorem 3. The inclusion functor

$$
i: \mathscr{S} \longrightarrow \mathscr{M} V^{w}
$$

induces a homotopy equivalence

$$
v S_{\bullet} \mathscr{S} \longrightarrow v S_{\bullet} \mathscr{M} V^{w} .
$$

Following the pattern of manipulations of cartesian squares given in Section 11 of [8, pages $210-218]$ one arrives at the following theorem on the structure of $K(R)$.

Theorem 4. There is a homotopy-commutative square

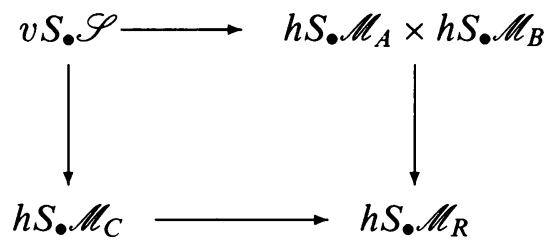

which is homotopy cartesian with respect to the simplicial homotopy which to any object of $\mathscr{S}$ associates its structure map $\kappa$.

In general, after geometric realization one can produce a splitting

$$
\left|v S_{\bullet} \mathscr{S}\right| \simeq\left|h S_{\bullet} \mathscr{M}_{C}\right| \times\left|h S_{\bullet} \mathscr{M}_{C}\right| \times \operatorname{Nil}\left(C ; A^{\prime}, B^{\prime}\right),
$$

where the third factor remains obscure in general. In case the ring $C$ is regular coherent in the sense of [8, page 160], one may also use the techniques of this paper to prove an analogue of Theorem 11.2 of [8] which identifies the homotopy type of $v S_{\bullet} \mathscr{S}$ with that of $h S_{0} \mathscr{M}_{C} \times h S_{\bullet} \mathscr{M}_{C}$. Then a little more manipulation leads to the familiar Mayer-Vietoris type sequence of algebraic $K$-groups:

$$
\cdots \longrightarrow K_{n+1}(R) \longrightarrow K_{n}(C) \longrightarrow K_{n}(A) \oplus K_{n}(B) \longrightarrow K_{n}(R) \longrightarrow \cdots
$$

Going beyond what we mention here, the proof of the equivalence of stable $K$-theory with topological Hochschild homology which we have outlined in [4] requires a partial analysis of the Nil-term for certain diagrams of $A_{\infty}$-rings. We establish a framework for doing this in [5], where, to warm up, we will return to the result of [8] just mentioned. With these remarks out of the way, we proceed to collect some background material, and then we move on to the proofs. 


\section{BASICS}

The categories of simplicial modules we use in this paper are defined in Section 2.3 of [9]. We start this section with a few amplifications of the ideas introduced there. Categories of simplicial modules without extra finiteness conditions have also been introduced in Chapter II, Sections 4 and 6, of [3].

Let $A$ be a ring with 1. A right module over $A$ is a simplicial abelian group $M$, together with a unital and associative action of $A$ from the right. This action may be codified in terms of a homomorphism of abelian groups $M \otimes A \longrightarrow M$ where the tensor product is taken degreewise, such that certain diagrams commute. The symbol $\mathscr{M}(A)$ denotes the category of right $A$-modules and their $A$-linear maps.

A simplicial set $Y$ gives rise to a module $[Y] A$ if we let

$$
([Y] A)_{n}=\left[Y_{n}\right] A,
$$

the free right $A$-module generated by $Y_{n}$. We may, occasionally, need to construct left modules in a similar manner. Notice that if $X=\Delta^{q} / \partial \Delta^{q}$ is a standard model for the simplicial $q$-sphere, then $[X] A$ is isomorphic to a product of simplicial Eilenberg-Mac Lane spaces $K(A, q) \times K(A, 0)$. If $Y$ is a simplicial set with a basepoint $*$, then one reduces $[Y] A$ by dividing out by the submodule $[*] A$. For example, if we view the sphere above as a pointed simplicial set, the construction produces a simplicial Eilenberg-Mac Lane space $K(A, q)$.

To attach an $n$-cell to an $A$-module $M$ means to form a pushout of the kind

$$
M \longleftarrow\left[\partial \Delta^{n}\right] A \longrightarrow\left[\Delta^{n}\right] A .
$$

A module $N$ is obtained from $M$ by attaching cells if it can be constructed from $M$ by means of this process together with taking a direct limit; we also observe that in this case $M \longrightarrow N$ is a free map in the sense of [3, page 6.3].

We will also be using the following notations. A pair of simplicial sets $\left(Y, Y^{\prime}\right)$ gives rise to a pair of modules denoted by $\left[Y, Y^{\prime}\right] A$. More generally, if $M$ is any $A$-module, then we define

$$
\left[Y, Y^{\prime}\right] M=\left[Y, Y^{\prime}\right] \mathbf{Z} \otimes M,
$$

where $\mathbf{Z}$ denotes the integers and where we take the tensor product degreewise.

The category of modules we are interested in is $\mathscr{M}_{f}(A)$, the full subcategory $\mathscr{M}(A)$ whose objects are the modules obtainable from the zero module by attaching finitely many cells. In this category the cofibrations are the free maps and the weak equivalences are the maps which become homotopy equiv. alences after realization. However, simplicial abelian groups are Kan sets, so it would suffice to use simplicial homotopy equivalences. Recall that the crucial axiom for cofibrations is that a cofibration admits cobase changes. That is, if $c: M \longrightarrow N$ is a cofibration and $f: M \longrightarrow M^{\prime}$ is arbitrary, then the pushout square

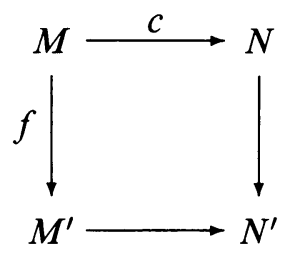


exists in $\mathscr{M}_{f}(A)$ and, most importantly, $M^{\prime} \longrightarrow N^{\prime}$ is also a cofibration. That this latter condition is satisfied may be verified by induction using the cell attachment characterization of a cofibration.

We make explicit the notion of cylinder functor [9, page 348] admitted by each of our categories of modules. In the category $\mathscr{M}_{f}(A)$, for instance, the mapping cylinder of a homomorphism $f: M \longrightarrow N$ is constructed by taking the following pushout:

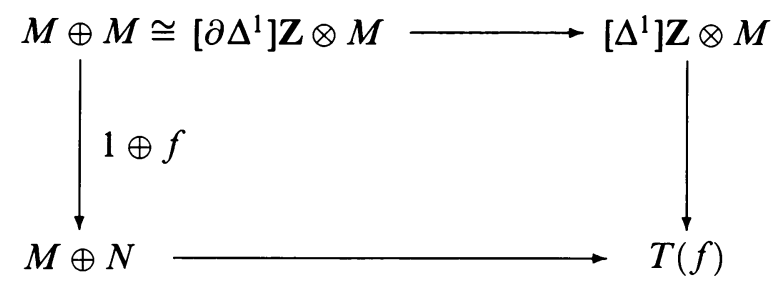

where the upper horizontal arrow is the cofibration induced by the inclusion of simplicial sets $\partial \Delta^{1} \longrightarrow \Delta^{1}$. We also note that the weak equivalences satisfy the extension and saturation axioms of [9, page 327], as well as the cylinder axiom [9, page 349].

As mentioned in the introduction, the category $\mathscr{M} V$ inherits notions of cofibration and mapping cylinder from the categories $\mathscr{M}_{f}(A), \mathscr{M}_{f}(B), \mathscr{M}_{f}(C)$, and $\mathscr{M}_{f}(R)$. A map

$$
\left(c, c_{A}, c_{B}, c_{C}\right):\left(M, M_{A}, M_{B}, M_{C}, l, \kappa\right) \longrightarrow\left(N, N_{A}, N_{B}, N_{C}, l, \kappa\right)
$$

is a cofibration if and only if each of the maps $c, c_{A}, c_{B}$, and $c_{C}$ is a cofibration in its module category. To see that the cobase-change axiom holds, let

$$
\left(f, f_{A}, f_{B}, f_{C}\right):\left(M, M_{A}, M_{B}, M_{C}, \imath, \kappa\right) \longrightarrow\left(M^{\prime}, M_{A}^{\prime}, M_{B}^{\prime}, M_{C}^{\prime}, l, \kappa\right)
$$

be an arbitrary map of Mayer-Vietoris presentations and define $N^{\prime}, N_{A}^{\prime}, N_{B}^{\prime}$, and $N_{C}^{\prime}$ via the pushout squares

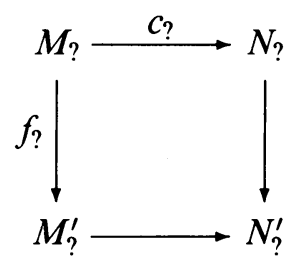

in the appropriate categories. Then the cofibration condition in the axiom is satisfied, but we need to know that the given maps $l$ and $\kappa$ induce an exact sequence

$$
0 \longrightarrow N^{\prime} \stackrel{\iota}{\longrightarrow} N_{A}^{\prime} \otimes_{A} R \oplus N_{B}^{\prime} \otimes_{B} R \stackrel{\kappa}{\longrightarrow} N_{C}^{\prime} \otimes_{C} R \longrightarrow 0
$$


For this, consider the following diagram.

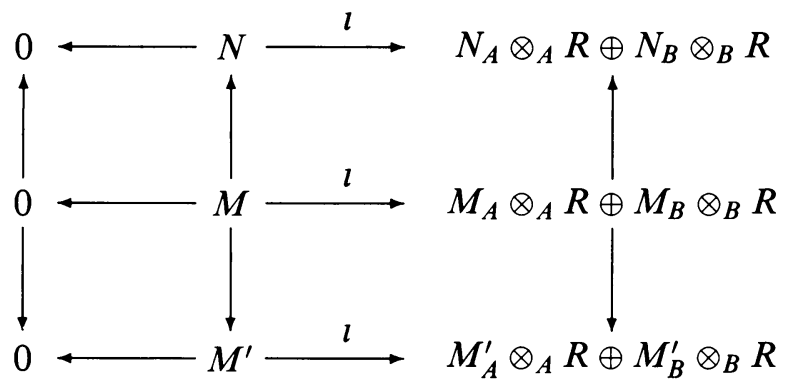

Now calculate the colimit iteratively in two ways, and recall that tensoring with $R$ is exact, by our freeness hypothesis, so that tensoring with $R$ then preserves colimits. Calculating the column colimits first, one finds that one expression for the colimit of the diagram is $\left(N_{A}^{\prime} \otimes_{A} R \oplus N_{B}^{\prime} \otimes_{B} R\right) / l\left(N^{\prime}\right)$, and, calculating the row colimits first, one finds that another expression for the colimit of the diagram is $N_{C}^{\prime} \otimes_{C} R$. It follows that

$$
0 \longrightarrow N^{\prime} \stackrel{l}{\longrightarrow} N_{A}^{\prime} \otimes_{A} R \oplus N_{B}^{\prime} \otimes_{B} R \stackrel{\kappa}{\longrightarrow} N_{C}^{\prime} \otimes_{C} R \longrightarrow 0
$$

is exact.

Similarly, we may introduce mapping cylinders on $\mathscr{M} V$ by pulling them back from the mapping cylinders on $\mathscr{M}_{f}(A), \mathscr{M}_{f}(B), \mathscr{M}_{f}(C)$, and $\mathscr{M}_{f}(R)$. One first observes that

$$
T\left(f_{A} \otimes_{A} R\right)=T\left(f_{A}\right) \otimes_{A} R
$$

and so on for the other cases. Then, since we have defined the mapping cylinders on our module categories in terms of pushouts, we derive from the argument above that taking mapping cylinders induces a Mayer-Vietoris presentation

$$
\left(T(f), T\left(f_{A}\right), T\left(f_{B}\right), T\left(f_{C}\right), l, \kappa\right)
$$

if $\left(f, f_{A}, f_{B}, f_{C}\right)$ is a map of Mayer-Vietoris presentations, and that the cofibration conditions [8, page 348] put on the front and back inclusions are satisfied.

Recall that we defined a map $\left(g, g_{A}, g_{B}, g_{C}\right)$ in $\mathscr{M} V$ to be a $w$-equivalence if $g$ is a weak homotopy equivalence and to be a $v$-equivalence if the three maps $g_{A}, g_{B}$, and $g_{C}$ are all weak homotopy equivalences. Since the weak homotopy equivalences in the module categories satisfy the extension, saturation, and cylinder axioms, it follows that both the $v$-equivalences and the $w$-equivalences satisfy these three axioms, too. Therefore Theorem 1.6.4 of [9, page 350] applies to the category $\mathscr{M} V$ with these notions of weak equivalence and we immediately derive the following proposition.

Proposition 2.1. Denote by $\mathscr{M} V^{w}$ the subcategory of $\mathscr{M} V$ consisting of MayerVietoris presentations in which the $R$-module is contractible. Then there is a fibration-up-to-homotopy

$$
v S_{\bullet} \mathscr{M} V^{w} \longrightarrow v S_{\bullet} \mathscr{M} V \longrightarrow w S_{\bullet} \mathscr{M} V
$$

We now prove the first of the identification theorems. It will be seen that the argument is basically formal and depends on another fundamental result of 
$K$-theory called the additivity theorem. See [9, page 336] and [1] for proofs and various formulations of the result and [6] for reductions of some other standard theorems in $K$-theory to applications of the additivity theorem.

Theorem 1. The forgetful functors from $\mathscr{M} V$ to the module categories $\mathscr{M}_{f}(A)$, $\mathscr{M}_{f}(B)$, and $\mathscr{M}_{f}(C)$ induce a homotopy equivalence

$$
u_{*}: v S_{\bullet} \mathscr{M} V \longrightarrow h S_{\bullet} \mathscr{M}_{f}(A) \times h S_{\bullet} \mathscr{M}_{f}(B) \times h S_{\bullet} \mathscr{M}_{f}(C) .
$$

Proof. Let

$$
u_{A}: \mathscr{M} V \longrightarrow \mathscr{M}_{f}(A)
$$

denote the functor which selects the module $M_{A}$ from a Mayer-Vietoris presentation

$$
0 \longrightarrow M \stackrel{\iota}{\longrightarrow} M_{A} \otimes_{A} R \oplus M_{B} \otimes_{B} R \stackrel{\kappa}{\longrightarrow} M_{C} \otimes_{C} R \longrightarrow 0,
$$

and define $u_{B}$ and $u_{C}$ similarly. Now the functor $u_{A}$ has an obvious section $s_{A}$ which associates to an $A$-module $M_{A}$ the Mayer-Vietoris presentation

$$
0 \longrightarrow\left(M_{A} \otimes_{A} R\right) \stackrel{=}{\longrightarrow} M_{A} \otimes_{A} R \oplus 0 \longrightarrow 0 \longrightarrow 0 .
$$

Define a section $s_{B}$ of $u_{B}$ similarly.

Define a section $s_{C}$ of $u_{C}$ by associating to the $C$-module $M_{C}$ the MayerVietoris presentation

$$
0 \longrightarrow M_{C} \otimes_{C} R \stackrel{\Delta}{\longrightarrow}\left(M_{C} \otimes_{C} A\right) \otimes_{A} R \oplus\left(M_{C} \otimes_{C} B\right) \otimes_{B} R \stackrel{\tilde{\Delta}}{\longrightarrow} M_{C} \otimes_{C} R \longrightarrow 0,
$$

where $\Delta$ is the diagonal and $\tilde{\Delta}$ is the skew codiagonal. The composites $u_{C} \circ s_{A}$, $u_{B} \circ s_{A}, u_{C} \circ s_{B}$, and $u_{A} \circ s_{B}$ are all trivial, the composite $u_{A} \circ s_{C}$ is the functor

$$
\alpha: \mathscr{M}_{f}(C) \longrightarrow \mathscr{M}_{f}(A)
$$

whose value on $M_{C}$ is $M_{C} \otimes_{C} A$, and the composite $u_{B} \circ s_{C}$ is a similar functor

$$
\beta: \mathscr{M}_{f}(C) \longrightarrow \mathscr{M}_{f}(B) .
$$

It follows that if we write

$$
s_{*}=\left(s_{A}\right)_{*} \vee\left(s_{B}\right)_{*} \vee\left(s_{C}\right)_{*}: h S_{0} \mathscr{M}_{f}(A) \times h S_{\bullet} \mathscr{M}_{f}(B) \times h S_{\bullet} \mathscr{M}_{f}(C) \longrightarrow v S_{\bullet} \mathscr{M} V,
$$

where $\vee$ indicates the $H$-space addition on $v S_{\mathscr{O}} \mathscr{M} V$ induced by the summing of Mayer-Vietoris presentations, then $u_{*} \circ s_{*}$ is a self-map of $h S_{*} \mathscr{M}_{f}(A) \times$ $h S_{0} \mathscr{M}_{f}(B) \times h S_{0} \mathscr{M}_{f}(C)$ which has the matrix representation

$$
\left(\begin{array}{ccc}
\mathrm{Id}_{*} & 0 & \alpha_{*} \\
0 & \mathbf{I d}_{*} & \beta_{*} \\
0 & 0 & \mathrm{Id}_{*}
\end{array}\right)
$$

and is therefore a self-equivalence. It follows that $u_{*}$ has a right homotopy inverse. 
To complete the proof, we have to prove that $u_{*}$ has a left homotopy inverse. This we will do using the additivity theorem. First note that, given a MayerVietoris presentation

$$
0 \longrightarrow M \stackrel{\iota}{\longrightarrow} M_{A} \otimes_{A} R \oplus M_{B} \otimes_{B} R \stackrel{\kappa}{\longrightarrow} M_{C} \otimes_{C} R \longrightarrow 0,
$$

the arrow $l$ defines a natural transformation

$$
\imath: \mathrm{Id} \longrightarrow s_{A} \circ u_{A} \oplus s_{B} \circ u_{B} .
$$

Explicitly, this is represented by the diagram

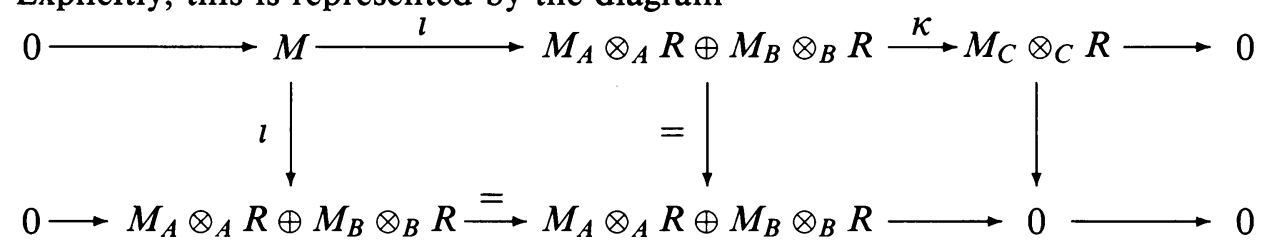

Unfortunately, the natural transformation is not a cofibration in the category $\mathscr{M} V$, but we take mapping cylinders to correct this, obtaining an exact functor $F$ whose value on our typical Mayer-Vietoris presentation is

$0 \longrightarrow T(l) \longrightarrow\left(\left[\Delta^{1}\right] \mathbf{Z} \otimes M_{A}\right) \otimes_{A} R \oplus\left(\left[\Delta^{1}\right] \mathbf{Z} \otimes M_{B}\right) \otimes_{B} R \longrightarrow C\left(M_{C}\right) \otimes_{C} R \longrightarrow 0$

where $C\left(M_{C}\right)=T\left(M_{C} \longrightarrow 0\right)$ is the cone on $M_{C}$. Now we have a factorization of $l$ through $F$,

$$
\mathrm{Id} \longrightarrow F \stackrel{\sim}{\longrightarrow} s_{A} \circ u_{A} \oplus s_{B} \circ u_{B},
$$

in which the transformation Id $\longrightarrow F$ is a cofibration, because it is the front inclusion of a mapping cylinder, and the transformation $F \longrightarrow s_{A} \circ u_{A} \oplus s_{B} \circ u_{B}$ is a $v$-equivalence, because the mapping cylinders for Mayer-Vietoris presentations satisfy the cylinder axiom. Applying Proposition 1.3.1 of [9, page 330], which is just an application to the $S_{\bullet}$ c construction of the principle that a natural transformation of functors induces a simplicial homotopy of maps of nerves of categories, it follows that $F$ and $s_{A} \circ u_{A} \oplus s_{B} \circ u_{B}$ induce homotopic maps on $v S_{\bullet} \mathscr{M} V$ :

$$
F_{*} \simeq\left(s_{A} \circ u_{A}\right)_{*} \vee\left(s_{B} \circ u_{B}\right)_{*} .
$$

Now we apply the additivity theorem $[9$, page 336$]$ to the cofibration sequence of functors

obtaining a homotopy

$$
\mathrm{Id} \longrightarrow F \longrightarrow F / \mathrm{Id}=F^{\prime},
$$

$F_{*} \simeq \mathrm{Id}_{*} \vee F_{*}^{\prime}$

of self-maps of $v S_{\mathscr{0}} \mathscr{M} V$.

One sees that the value of $F^{\prime}$ on a Mayer-Vietoris presentation

$$
0 \longrightarrow M \stackrel{\iota}{\longrightarrow} M_{A} \otimes_{A} R \oplus M_{B} \otimes_{B} R \stackrel{\kappa}{\longrightarrow} M_{C} \otimes_{C} R \longrightarrow 0
$$

is the Mayer-Vietoris presentation

$$
\begin{aligned}
0 \longrightarrow & T(l) / M \longrightarrow\left(\frac{\left[\Delta^{1}\right] \mathbf{Z} \otimes M_{A}}{[0] \mathbf{Z} \otimes M_{A}}\right) \\
& \otimes_{A} R \oplus\left(\frac{\left[\Delta^{1}\right] \mathbf{Z} \otimes M_{B}}{[0] \mathbf{Z} \otimes M_{B}}\right) \otimes_{B} R \longrightarrow B\left(M_{C}\right) \otimes_{C} R \longrightarrow 0,
\end{aligned}
$$


where $B\left(M_{C}\right)=C\left(M_{C}\right) / M_{C}$ is the classifying space, or reduced suspension of $M_{C}$, and the modules in the middle are contractible.

Now we want to relate $F^{\prime}$ to $s_{C} \circ u_{C}$. Recall from Section 1 that we have written the map $\kappa$ in the original Mayer-Vietoris presentation as $\kappa=\kappa_{\alpha}-\kappa_{\beta}$ where $\kappa_{\alpha}\left(M_{A}\right) \subset M_{C} \otimes_{C} A$ and $\kappa_{\beta}\left(M_{B}\right) \subset M_{C} \otimes_{C} B$. By exactness $\kappa_{\alpha} \circ l=$ $\kappa_{\beta} \circ l$, so we may use these two maps to define a natural transformation

$$
\text { Id } \longrightarrow s_{C} \circ u_{C} \text {. }
$$

If we apply $F^{\prime}$ to this natural transformation, we obtain a ladder diagram

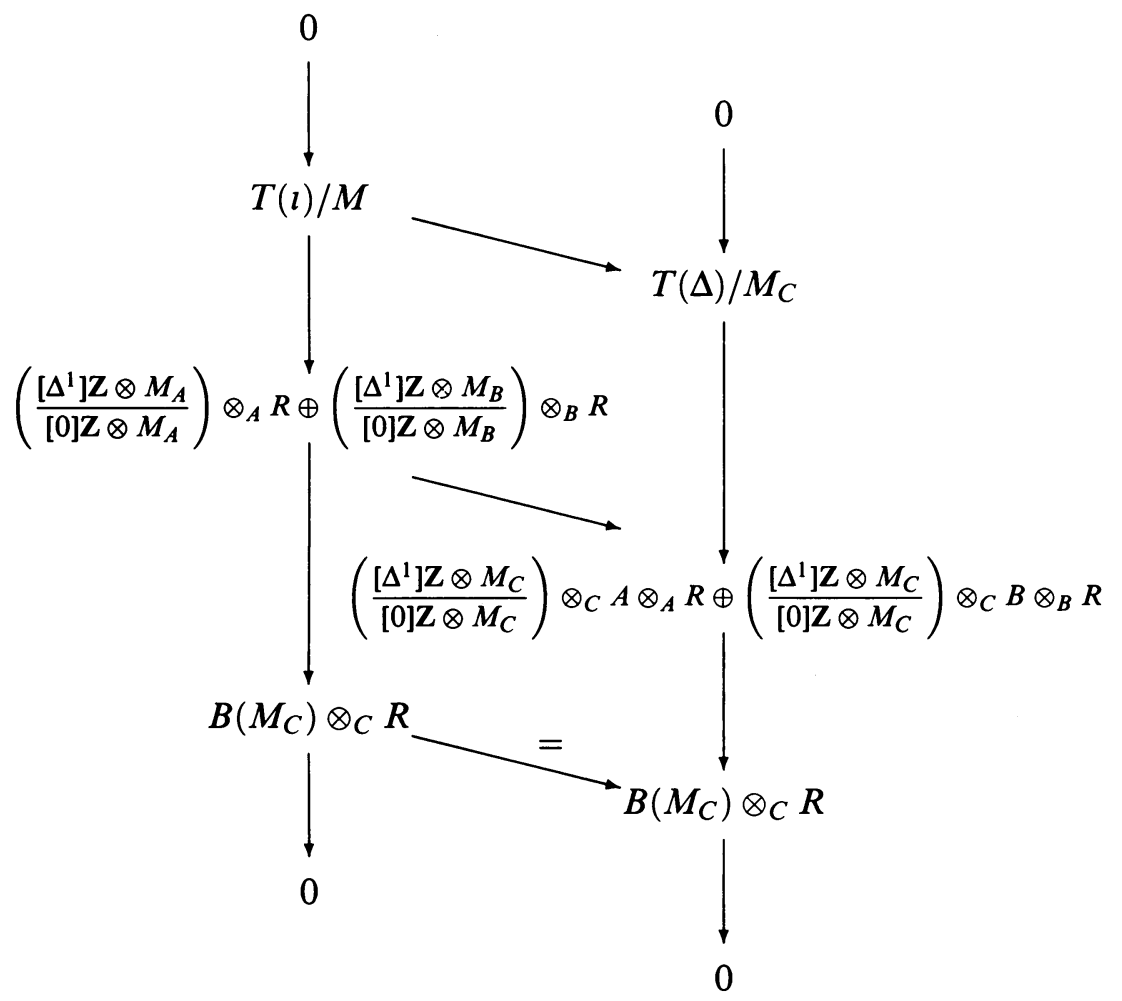

Applying the five lemma to the ladder, we see that the diagram displays a $v$ equivalence of Mayer-Vietoris presentations, so that on the space $\left|v S_{\bullet} \mathscr{M} V\right|$ we have $F_{*}^{\prime} \simeq\left(F^{\prime} \circ s_{C} \circ u_{C}\right) *$.

To summarize what we have learned so far, we have

$$
\left(s_{A} \circ u_{A}\right)_{*} \vee\left(s_{B} \circ u_{B}\right)_{*} \simeq F_{*} \simeq \operatorname{Id}_{*} \vee F_{*}^{\prime} \simeq \operatorname{Id}_{*} \vee\left(F^{\prime} \circ s_{C} \circ u_{C}\right)_{*}
$$

Recall that $h S_{\circ} \mathscr{M}(C)_{f}$ is a connected $H$-space with respect to $\mathrm{V}$, and that suspension induces a homotopy inverse with respect to the $H$-space operation [9, page 349]. Therefore, we may shift $\left(F^{\prime} \circ s_{C} \circ u_{C}\right)_{*}$ to the other side of the homotopy, obtaining finally

$$
\left(s_{A} \circ u_{A}\right)_{*} \vee\left(s_{B} \circ u_{B}\right)_{*} \vee\left(F^{\prime} \circ s_{C} \circ(-1) \circ u_{C}\right)_{*} \simeq \mathbf{I d}_{*}
$$

It follows that $u_{*}$ has a left homotopy inverse, as required. 
Concerning this proof we make two remarks. First, we invite the reader who likes to play with functors to verify the formula

$$
\left(F^{\prime} \circ s_{C}\right)_{*} \simeq\left(s_{A} \circ \alpha\right)_{*} \vee\left(s_{B} \circ \beta\right)_{*} .
$$

Second, we call attention to the fact that this proof is purely $K$-theoretical, making use only of functors built into the definitions of the diagram categories we are using, mapping cylinders, and the additivity theorem. We will see in [5] that essentially the same argument proves a parallel result for simplicial rings, once the appropriate definitions are made.

\section{APPROXIMATION THEOREM}

Now we recall the approximation theorem (Theorem 1.6.7 on page 354 of [9]). This theorem gives conditions under which an exact functor between two categories with cofibrations and weak equivalences induces an equivalence at the level of the $K$-theories of the two categories. Roughly speaking, if the functor passes to an equivalence of homotopy categories, then it induces an equivalence of the $K$-theories.

Specifically, let $F: \mathscr{A} \longrightarrow \mathscr{B}$ be an exact functor of categories with cofibrations and weak equivalences. One says that $F$ has the approximation property if it satisfies these two conditions:

App 1: An arrow in $\mathscr{A}$ is a weak equivalence if and only if its image in $\mathscr{B}$ is a weak equivalence.

App 2: Given any object $A$ in $\mathscr{A}$ and any map $x: F(A) \longrightarrow$ $B$ in $\mathscr{B}$, there exist a cofibration $a: A \longrightarrow A^{\prime}$ and a weak equivalence $x^{\prime}: F\left(A^{\prime}\right) \longrightarrow B$ in $\mathscr{B}$ such that the following triangle commutes:

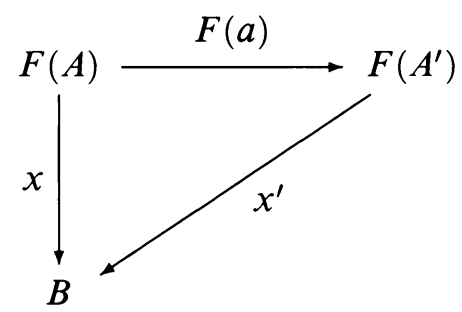

We may now state the approximation theorem. (Compare [9, page 354].)

Theorem 5. Let $\mathscr{A}$ and $\mathscr{B}$ be categories with cofibrations and weak equivalences. Suppose that the weak equivalences in $\mathscr{A}$ and $\mathscr{B}$ satisfy the saturation axiom. Suppose further that $\mathscr{A}$ has a cylinder functor and that weak equivalences in $\mathscr{A}$ satisfy the cylinder axiom. Let $F: \mathscr{A} \longrightarrow \mathscr{B}$ be an exact functor and suppose that $F$ has the approximation property. Then the induced maps $w \mathscr{A} \longrightarrow w \mathscr{B}$ and $w S_{\bullet} \mathscr{A} \longrightarrow w S_{\bullet} \mathscr{B}$ are homotopy equivalences.

Before we can get to our applications of the theorem, we state the technical results which are used to verify the difficult condition App 2. To prove Theorem 2 concerning the forgetful functor $u_{R}: \mathscr{M} V \longrightarrow \mathscr{M}_{f}(R)$, we use the following fact. 
Proposition 3.1. Given a Mayer-Vietoris presentation pair

$$
\begin{gathered}
0 \longrightarrow\left(M, M^{\prime}\right) \stackrel{l}{\longrightarrow}\left(M_{A}, M_{A}^{\prime}\right) \otimes_{A} R \oplus\left(M_{B}, M_{B}^{\prime}\right) \otimes_{B} R \\
\stackrel{\kappa}{\longrightarrow}\left(M_{C}, M_{C}^{\prime}\right) \otimes_{C} R \longrightarrow 0
\end{gathered}
$$

and a pair of finite simplicial sets $\left(X, X^{\prime}\right)$ together with a pair of maps of simplicial $R$-modules

$$
\left(f, f^{\prime}\right):\left[X, X^{\prime}\right] R \longrightarrow\left(M, M^{\prime}\right)
$$

then there is a Mayer-Vietoris presentation

$$
0 \longrightarrow R \stackrel{\iota}{\longrightarrow} F_{A} \otimes_{A} R \oplus F_{B} \otimes_{B} R \stackrel{\kappa}{\longrightarrow} F_{C} \otimes_{C} R \longrightarrow 0
$$

of the free $R$-module of rank one, where $F_{A}, F_{B}$, and $F_{C}$ are also free over their respective rings, and an extension of $\left(f, f^{\prime}\right)$ to a pair of maps of Mayer-Vietoris presentations, as follows.

$$
\begin{array}{ccccc}
{\left[X, X^{\prime}\right] R} & \longrightarrow & {\left[X, X^{\prime}\right] F_{A} \otimes_{A} R \oplus\left[X, X^{\prime}\right] F_{B} \otimes_{B} R} & \longrightarrow & {\left[X, X^{\prime}\right] F_{C} \otimes_{C} R} \\
\downarrow & & & & \\
\downarrow & & & \\
\left(M, M^{\prime}\right) & \stackrel{\iota}{\longrightarrow} & \left(M_{A}, M_{A}^{\prime}\right) \otimes_{A} R \oplus\left(M_{B}, M_{B}^{\prime}\right) \otimes_{B} R & \stackrel{\kappa}{\longrightarrow} & \left(M_{C}, M_{C}^{\prime}\right) \otimes_{C} R
\end{array}
$$

We have omitted the zeroes at the beginning and end of each row in the preceding diagram to save space. Also, in all our applications $f^{\prime}$ will be the zero map, but a little extra generality does not hurt.

Proof. The result is easily established by induction up the skeleta of the pair, taking direct sums of Mayer-Vietoris presentations constructed using Lemma 3.3 stated below. Note that this lemma is available only for genuine rings, so it is at this point a restriction in the range of our results appears.

To state Lemma 3.3, we need to refer the reader to parts of section one of [8]. First recall the recipe

$R=C \oplus A^{\prime} \oplus B^{\prime} \oplus A^{\prime} \otimes_{C} B^{\prime} \oplus B^{\prime} \otimes_{C} A^{\prime} \oplus A^{\prime} \otimes_{C} B^{\prime} \otimes_{C} A^{\prime} \oplus B^{\prime} \otimes_{C} A^{\prime} \otimes_{C} B^{\prime} \oplus \cdots$

from the introduction and write $A_{n}^{\prime}$ for the summand which has $n$ factors and $A^{\prime}$ on the left. Define $B_{n}^{\prime}$ similarly, so that

$$
R=C \oplus \cdot\left(\bigoplus_{n \geq 1} A_{n}^{\prime}\right) \oplus\left(\bigoplus_{n \geq 1} B_{n}^{\prime}\right) \text {. }
$$

If we define $A_{0}^{\prime}=B_{0}^{\prime}=C$, then we have $A^{\prime} \otimes_{C} B_{n}^{\prime}=A_{n+1}^{\prime}$ and $B^{\prime} \otimes_{C} A_{n}^{\prime}=B_{n+1}^{\prime}$ for $n \geq 0$. Writing $A^{\prime \prime}=\bigoplus_{n \geq 1} A_{n}^{\prime}$ and $B^{\prime \prime}=\bigoplus_{n \geq 1} B_{n}^{\prime}$, we can put the recipe in the form

$$
R=C \oplus A^{\prime \prime} \oplus B^{\prime \prime} .
$$

Using the splittings $A=C \oplus A^{\prime}$ and $B=C \oplus B^{\prime}$ and collecting differently, we see that we can also write

$$
R=A \oplus A \otimes_{C} B^{\prime \prime} \quad \text { and } \quad R=B \oplus B \otimes_{C} A^{\prime \prime} .
$$

If we now choose 1 as the basis element for $C$ and $C$-bases for $A^{\prime}$ and $B^{\prime}$, for which we write $\langle C\rangle,\left\langle A^{\prime}\right\rangle$, and $\left\langle B^{\prime}\right\rangle$, respectively, then these choices induce 
$C$-bases $\left\langle A_{n}^{\prime}\right\rangle,\left\langle A^{\prime \prime}\right\rangle,\left\langle B_{n}^{\prime}\right\rangle$, and $\left\langle B^{\prime \prime}\right\rangle$, respectively. Then a $C$-basis for $R$ may be written

$$
\langle R\rangle=\langle C\rangle \cup\left\langle A^{\prime \prime}\right\rangle \cup\left\langle B^{\prime \prime}\right\rangle .
$$

Looking at the description $R=A \oplus A \otimes_{C} B^{\prime \prime}$, we find a left $A$-basis for $R$, which we denote $T_{A}$ and which is $\langle C\rangle \cup\left\langle B^{\prime \prime}\right\rangle$ when viewed as a subset of $R$. Similarly we find and identify a left $B$-basis $T_{B}$. In [8] the three sets $T_{A}, T_{B}$, and $\langle R\rangle$ are organized into a directed tree $T$ with a basepoint. The disjoint union of the $A$-basis $T_{A}$ and the $B$-basis $T_{B}$ forms the set of vertices and the $C$-basis $\langle R\rangle$ forms the set of edges of $T$. Notice that the multiplicative identity in $R$ appears in three roles: as $1_{C} \in\langle R\rangle$, as $1_{A} \in T_{A}$, and as $1_{B} \in T_{B}$. We have defined the bases in such a way that there are a bijection $\langle A\rangle \times T_{A} \longrightarrow\langle R\rangle$, written $(a, x) \mapsto a \cdot x$ on an element of the cartesian product, and a similar bijection $\langle B\rangle \times T_{B} \longrightarrow\langle R\rangle$. Both of these are used in [8] to define incidence and to give orientation to the edges.

An alternative inductive description of the tree $T$ as a tree filtered by subtrees follows. Let $T_{0}$ consist of the vertices $1_{A} \in T_{A}$ and $1_{B} \in T_{B}$ connected by the edge $1_{C} \in\langle R\rangle$. We make the arbitrary choice that the edge is oriented from $1_{A}$ to $1_{B}$. This then fixes the vertex $1_{A}$ as the basepoint, or root. Proceeding inductively, suppose that we have defined an increasing union

$$
T_{0} \subset T_{1} \subset \cdots \subset T_{n},
$$

so that the extremal vertices of $T_{n}$ are the disjoint union of $\left\langle A_{n}^{\prime}\right\rangle \subset T_{B}$ and $\left\langle B_{n}^{\prime}\right\rangle \subset T_{A}$. We now want to extend $T_{n}$ by adding and connecting the vertices $\left\langle A_{n+1}^{\prime}\right\rangle$ and $\left\langle B_{n+1}^{\prime}\right\rangle$. To see how to do this, we use the formulas $A^{\prime} \otimes_{C} B_{n}^{\prime}=A_{n+1}^{\prime}$ and $B^{\prime} \otimes_{C} A_{n}^{\prime}=B_{n+1}^{\prime}$ for $n \geq 0$ given above. For $a \in\left\langle A^{\prime}\right\rangle$ and $x \in\left\langle B_{n}^{\prime}\right\rangle$ we connect the vertex $x$ to the vertex $a x \in\left\langle A_{n+1}^{\prime}\right\rangle$ by a segment $a \cdot x \in\langle R\rangle$. The segment is oriented so as to run from $x$ to $a x$. The following figure illustrates the inclusions $T_{0} \subset T_{1} \subset T_{2}$.

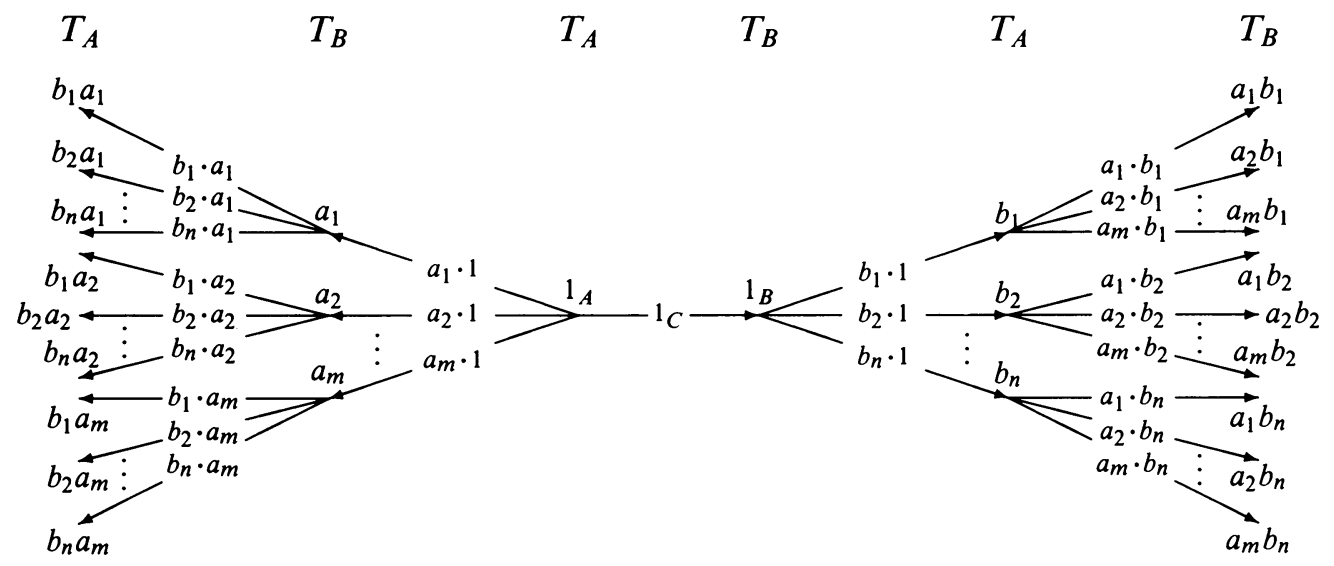

In the context of Mayer-Vietoris presentations of ordinary, i.e., not simplicial, modules we have the following version of Proposition 1.1 of [8].

Proposition 3.2. Let $N$ be the free right $R$-module on the basis element $n$. Let $\Delta$ be a finite subtree of $T$, containing the basepoint. Then $\Delta$ determines $a$ 
canonical Mayer-Vietoris presentation of $N$,

$$
0 \longrightarrow N \stackrel{\iota}{\longrightarrow} N_{A} \otimes_{A} R \oplus N_{B} \otimes_{B} R \stackrel{\kappa}{\longrightarrow} N_{C} \otimes_{C} R \longrightarrow 0,
$$

in which the modules $N_{A}, N_{B}$, and $N_{C}$ are also free. We denote this presentation by $\langle N, n, \Delta\rangle$. Also, given $m \in M$, there exists a map of $\langle N, n, \Delta\rangle$ into the Mayer-Vietoris presentation

$$
0 \longrightarrow M \stackrel{\iota}{\longrightarrow} M_{A} \otimes_{A} R \oplus M_{B} \otimes_{B} R \stackrel{\kappa}{\longrightarrow} M_{C} \otimes_{C} R \longrightarrow 0
$$

inducing $n \rightarrow m$ if and only if $\Delta$ contains a certain finite tree $\Delta(m)$. The entire map is uniquely determined by $m$.

One sees immediately that if one defines a category of such Mayer-Vietoris presentations $\langle N, n, \Delta\rangle$ over a fixed Mayer-Vietoris presentation pointed by $m \in M$, then the category has a terminal object, corresponding to the tree $\Delta(m)$, and that finite limits in the category may be constructed by taking the Mayer-Vietoris presentation corresponding to the union of the subtrees of $T$ occuring in the constituents of a diagram. Using these remarks, it is now easy to prove the following lemma.

Lemma 3.3. Suppose that

$$
0 \longrightarrow M \stackrel{\iota}{\longrightarrow} M_{A} \otimes_{A} R \oplus M_{B} \otimes_{B} R \stackrel{\kappa}{\longrightarrow} M_{C} \otimes_{C} R \longrightarrow 0
$$

is a given Mayer-Vietoris presentation of ordinary modules and that $\left\langle N_{i}, n_{i}, \Delta_{i}\right\rangle$, $1 \leq i \leq p$, is a family of Mayer-Vietoris presentations mapping to the given presentation, with $n_{i} \rightarrow m_{i}$. Then there is a single Mayer-Vietoris presentation of the free module on the generator $n,\langle N, n, \Delta\rangle$, admitting $p$ maps to the given presentation, such that the ith map carries $n$ to $m_{i}$.

Proof. We put $\Delta=\bigcup_{i=1}^{p} \Delta_{i}$, a subtree of $T$, and construct the corresponding Mayer-Vietoris presentation. By construction $\Delta$ contains $\Delta\left(m_{i}\right)$ for each $i$, so by Proposition 3.2 the desired maps exist.

Consequently, there is a standard Mayer-Vietoris presentation of the free $R$-module of rank $p$ mapping to the given presentation such that preferred generators are mapped respectively to the elements $m_{1}, \ldots, m_{p}$ of $M$.

In Section 5 we verify App 2 for the inclusion functor $i: \mathscr{S} \longrightarrow \mathscr{M} V^{w}$ using the following result.

Proposition 3.4. Let

$$
\left(0, M_{A}, M_{B}, M_{C}\right) \longrightarrow\left(N, N_{A}, N_{B}, N_{C}\right)
$$

be a cofibration of a split module into a Mayer-Vietoris presentation in which $N$ is contractible. Let $n \geq 0$ and let

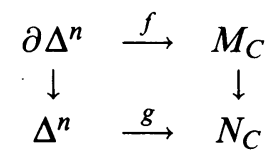

be a commutative diagram of simplicial maps. Then there is a homotopy of $g$ relative to the boundary to a map $g^{\prime}$, a free split (ordinary) module $\left(0, F_{A}\right.$, 
$\left.F_{B}, F_{C}\right)$, and maps of Mayer-Vietoris presentations

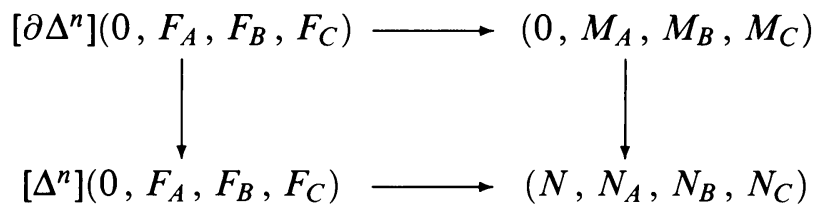

such that the diagram commutes and $g^{\prime}\left(\Delta^{n}\right) \subset \operatorname{Im}\left(F_{C} \otimes_{C} R\right)$.

Also, if $f$ maps a horn $\Lambda \subset \partial \Delta^{n}$ to zero, then we may assume that the restriction to $[\Lambda]\left(0, F_{A}, F_{B}, F_{C}\right)$ of the upper horizontal map in the diagram is also zero.

The tools for proving the lemma are some simplicial homotopy theory and the following version of Proposition 1.2 of [8, page 144].

First we recall the based augmented trees $+\Delta$ of $[8$, page 144]. One way to think of these objects is to consider the directed graph $T-\left\{1_{C}\right\}$, which consists of two trees. We may define an $A$-tree to be a finite subtree of $T-\left\{1_{C}\right\}$ with its base point at $1_{A}$. A $B$-tree is defined similarly. If $\Delta$ is an $A$ - or a $B$-tree, then we call $+\Delta=\Delta \cup\left\{1_{C}\right\}$ a based augmented tree, with the extra edge $1_{C}$ being called the augmentation segment. We write ${ }_{A} \Delta$ for a typical based augmented $A$-tree and ${ }_{B} \Delta$ for a based augmented $B$-tree.

Proposition 3.5. To any finite based augmented tree ${ }^{+} \Delta$ there is canonically associated a split module $\left\langle{ }^{+} \Delta\right\rangle$ of the form

$$
0 \longrightarrow 0 \longrightarrow F_{A} \otimes_{A} R \oplus F_{B} \otimes_{B} R \stackrel{\cong}{\longrightarrow} F_{C} \otimes_{C} R \longrightarrow 0
$$

in which $F_{A}, F_{B}$, and $F_{C}$ are free. Given a Mayer-Vietoris presentation of ordinary modules

$$
0 \longrightarrow M \stackrel{l}{\longrightarrow} M_{A} \otimes_{A} R \oplus M_{B} \otimes_{B} R \stackrel{\kappa}{\longrightarrow} M_{C} \otimes_{C} R \longrightarrow 0
$$

and an element $m^{\prime} \in M_{C}$, then there exist finite based augmented trees ${ }_{A} \Delta$ and ${ }_{B} \Delta$ and a map from the direct sum of the split modules $\left\langle{ }_{A} \Delta\right\rangle \oplus\left\langle{ }_{B} \Delta\right\rangle$ to the given Mayer-Vietoris presentation such that $m^{\prime}$ is contained in the image.

Although the argument in [8] for this result is not presented as such, it is actually an algorithm for constructing from an equation

$$
\kappa\left(\sum_{x \in T_{A}} m_{x} \otimes x, \sum_{y \in T_{B}} m_{y} \otimes y\right)=m^{\prime}
$$

based augmented trees ${ }_{A} \Delta$ and ${ }_{B} \Delta$ and the required map into the MayerVietoris presentation. By means of this algorithm we are provided with just enough naturality to produce the simplicial map we are looking for. That all our basic data is generated by one simplex is essential. To prove that the restriction to the horn of the map we construct is trivial also requires an analysis based on the algorithm. We therefore recommend that the reader review the proof of Proposition 3.5 in [8] with these points in mind. The reader may also find it helpful to refer to our picture of part of the tree $T$ above while doing so.

We also remark that Proposition 3.5 concerns maps into Mayer-Vietoris presentations, whereas Proposition 1.2 of [8] addresses a more general situation. 
Proof. [Proof of 3.4.] The case $n=0$ of 3.4 is a trivial consequence of the statement of Proposition 3.5 and no homotopy is required; we explain this in the course of the proof of Theorem 3 .

Now suppose $n \geq 1$. First use the isomorphism $M_{A} \otimes_{A} R \oplus M_{B} \otimes_{B} R \cong$ $M_{C} \otimes_{C} R$ and the given map of the split module into the Mayer-Vietoris presentation to produce

$$
f_{1}: \partial \Delta^{n} \longrightarrow N_{A} \otimes_{A} R \oplus N_{B} \otimes_{B} R
$$

lifting the composite map

$$
\partial \Delta^{n} \longrightarrow M_{C} \otimes_{C} R \longrightarrow N_{C} \otimes_{C} R .
$$

On the other hand,

$$
0 \longrightarrow N \stackrel{\iota}{\longrightarrow} N_{A} \otimes_{A} R \oplus N_{B} \otimes_{B} R \stackrel{\kappa}{\longrightarrow} N_{C} \otimes_{C} R \longrightarrow 0
$$

is a fibration and $\Lambda \subset \Delta^{n}$ is a trivial cofibration, so we may pick a lifting

$$
F_{0}: \Delta^{n} \longrightarrow N_{A} \otimes_{A} R \oplus N_{B} \otimes_{B} R
$$

of the map $\Delta^{n} \longrightarrow N_{C} \otimes_{C} R$ extending $f_{1} \mid \Lambda$. Put $f_{0}=F_{0} \mid \partial \Delta^{n}$. By construction $f_{1}-f_{0}$ is a map to the fibre $N$, which is contractible and a Kan set, so that $f_{1}-f_{0}$ is simplicially null homotopic inside of $N$ by a homotopy which is zero on $\Lambda$. Translating the homotopy, $f_{1}$ and $f_{0}$ are simplicially fibrewise homotopic relative to $\Lambda$. Paste the homotopy onto $\Delta^{n}$ to obtain

$$
\Delta^{n} \cup\left(\partial \Delta^{n} \times \Delta^{1}\right) \longrightarrow N_{A} \otimes_{A} R \oplus N_{B} \otimes_{B} R
$$

extending $F_{0}$ and extend this map to

$$
H: \Delta^{n} \times \Delta^{1} \longrightarrow N_{A} \otimes_{A} R \oplus N_{B} \otimes_{B} R
$$

by the Kan extension property. Let $F_{1}=H \mid \Delta^{n} \times 1$ and put $g^{\prime}=\kappa \circ F_{1}$. We have a commutative diagram

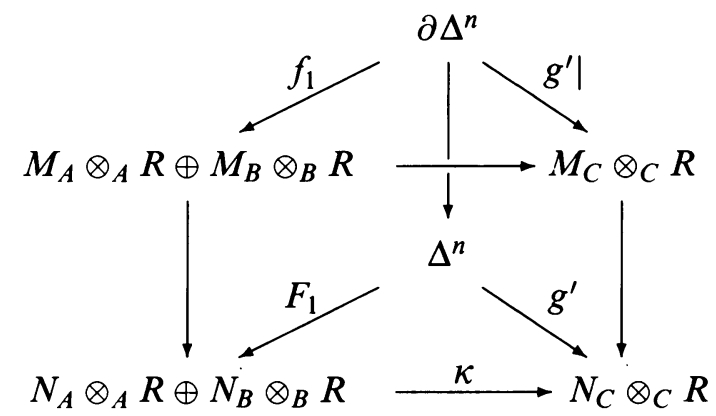

and, by construction, $g^{\prime}$ is homotopic relative to the boundary to the original map $g$. Moreover, if $g^{\prime}|\Lambda=g| \Lambda$ is the zero map, then $F_{1} \mid \Lambda$ is also zero, since $M_{A} \otimes_{A} R \oplus M_{B} \otimes_{B} R \longrightarrow M_{C} \otimes_{C} R$ is an isomorphism.

Now we can apply the algorithm described on page 145 of [8] to the lifting

$$
F_{1}\left(\sigma^{n}\right)=\left(\sum_{x \in T_{A}} n_{x} \otimes x, \sum_{y \in T_{B}} n_{y} \otimes y\right)
$$


of $g^{\prime}\left(\sigma^{n}\right), \sigma^{n}$ being the generating simplex of $\Delta^{n}$. We obtain a split module $\left(0, F_{A}, F_{B}, F_{C}\right)$ and a map to the $n$-simplices of $\left(N, N_{A}, N_{B}, N_{C}\right)$ with $g^{\prime}\left(\sigma^{n}\right)$ in the image. We must now extend the split module $\left(0, F_{A}, F_{B}, F_{C}\right)$ to a simplicial split module $\left[\Delta^{n}\right]\left(0, F_{A}, F_{B}, F_{C}\right)$, and we must also extend the map. For this we require the naturality implied by using the proof of Proposition 3.5 as described in [8] as an algorithm. Now, for any order-preserving map $\alpha:[k] \longrightarrow[n]$,

$$
\alpha^{*} F_{1}\left(\sigma^{n}\right)=\left(\sum \alpha^{*}\left(n_{x}\right) \otimes x, \sum \alpha^{*}\left(n_{y}\right) \otimes y\right)
$$

lifts $\alpha^{*} g^{\prime}\left(\sigma^{n}\right)$. Application of naturality to these liftings yields a simplicial map of Mayer-Vietoris presentations

$$
\left[\Delta^{n}\right]\left(0, F_{A}, F_{B}, F_{C}\right)=\bigoplus_{k} \bigoplus_{\alpha:[k] \longrightarrow[n]}\left(0, F_{A}, F_{B}, F_{C}\right) \longrightarrow\left(N, N_{A}, N_{B}, N_{C}\right)
$$

with $g^{\prime}\left(\Delta^{n}\right)$ in the image. We see that the restriction to $\left[\partial \Delta^{n}\right]\left(0, F_{A}, F_{B}, F_{C}\right)$ factors through the split module $\left(0, M_{A}, M_{B}, M_{C}\right)$ by making use of the preceding diagram.

To conclude, we need to see that if $g^{\prime}(\Lambda)=g(\Lambda)=0$, then $[\Lambda]\left(0, F_{A}, F_{B}\right.$, $\left.F_{C}\right)$ also maps to zero. This actually requires a look at the algorithm itself. Recall that we have seen earlier that the hypothesis implies $F_{1}(\Lambda)=0$, so that $[\Lambda] F_{A}$ and $[\Lambda] F_{B}$ map to zero, by following the algorithm. To see that $[\Lambda] F_{C}$ maps to zero, let $\left(0, \gamma_{A}, \gamma_{B}, \gamma_{C}\right)$ be the components of the map $[\Delta]\left(0, F_{A}, F_{B}, F_{C}\right) \longrightarrow\left(N, N_{A}, N_{B}, N_{C}\right)$. Now let $s$ be an edge of one of the trees ${ }_{A} \Delta$ or ${ }_{B} \Delta$, and suppose $S$ is extremal, incident with a terminal vertex $x \in T_{A}$, say. By the definition of the incidence relation in $T$ and the orientation of the tree $T, s=1 \otimes x$. Consequently, by definition of $\kappa, \kappa\left(n_{x}\right)=n_{s}$, so that

$$
\gamma_{C}\left(\Lambda \cdot n_{s}\right)=\gamma_{C}\left(\kappa \Lambda \cdot n_{x}\right)=\kappa\left(\gamma_{A} \Lambda \cdot n_{x}\right)=\kappa(0)=0
$$

Now suppose that $s$ is no longer an extremal edge, but that we know that $\gamma_{C}\left(\Lambda \cdot n_{s^{\prime}}\right)=0$ for all edges $s^{\prime}$ closer to a terminal vertex. Suppose again that the terminal vertex is $x \in T_{A}$, and let $x$ be the initial vertex of edges $s_{i}=a_{i} \otimes x$. By the algorithm $\kappa\left(n_{x}\right)=n_{s}+\sum n_{s_{i}} \otimes a_{i}$, so that

$$
\begin{aligned}
0 & =\kappa \gamma_{A}\left(\Lambda \cdot n_{x}\right)=\left(\gamma_{C} \otimes A\right) \kappa\left(\Lambda \cdot n_{x}\right) \\
& =\left(\gamma_{C} \otimes A\right)\left(\Lambda \cdot n_{s} \otimes 1+\sum \Lambda \cdot n_{s_{i}} \otimes a_{i}\right) \\
& =\gamma_{C}\left(\Lambda \cdot n_{s}\right) \otimes 1+\sum \gamma_{C}\left(\Lambda \cdot n_{s_{i}}\right) \otimes a_{i} \\
& =\gamma_{C}\left(\Lambda \cdot n_{s}\right) \otimes 1,
\end{aligned}
$$

by inductive hypothesis. Of course, similar arguments go through if we have vertices in $T_{B}$ involved.

In both of the following sections we make use of the following well-known lemma. A convenient reference is [2, page 369].

Lemma 3.6. Let

$$
0 \rightarrow Z_{k} \rightarrow C_{k} \rightarrow C_{k-1} \rightarrow \cdots \rightarrow C_{0} \rightarrow 0
$$


be an exact sequence of modules in which $C_{j}$ is free and finitely generated for all $j$. Then $Z_{k}$ is stably-free and finitely generated.

\section{Coarse equivalences AND $R$-MOdules}

In this section we take up the identification of the $K$-theory of the MayerVietoris presentations with respect to the coarse notion of weak equivalences.

Theorem 2. The forgetful functor

$$
u_{R}: \mathscr{M} V \longrightarrow \mathscr{M}_{f}(R)
$$

induces a homotopy equivalence

$$
w S_{\bullet} \mathscr{M} V \longrightarrow h S_{\bullet} \mathscr{M}_{f}(R) .
$$

Proof. All the categorical hypotheses of Theorem 5 (the approximation theorem) are satisfied by $\mathscr{M} V$ and $\mathscr{M}_{f}(R)$, where we take $w$-equivalences in $\mathscr{M} V$. We have to verify that $u_{R}$ has the approximation property. Condition App 1 is obviously satisfied so we must concern ourselves with the factorization condition App 2.

Here we are given an arbitrary Mayer-Vietoris presentation

$$
0 \longrightarrow M \stackrel{\iota}{\longrightarrow} M_{A} \otimes_{A} R \oplus M_{B} \otimes_{B} R \stackrel{\kappa}{\longrightarrow} M_{C} \otimes_{C} R \longrightarrow 0
$$

and an arbitrary $R$-map

$$
f: M \longrightarrow N,
$$

and we must construct a cofibration of Mayer-Vietoris presentations

$$
(c, \ldots):\left(M, M_{A}, M_{B}, M_{C}, l, \kappa\right) \longrightarrow\left(M^{\prime}, M_{A}^{\prime}, M_{B}^{\prime}, M_{C}^{\prime}, l, \kappa\right)
$$

and a homotopy equivalence

$$
f^{\prime}: M^{\prime} \longrightarrow N
$$

so that

$$
f^{\prime} \circ c=f
$$

If the map

$$
f_{*}: \pi_{0} M \longrightarrow \pi_{0} N
$$

is not onto, then we may find a constant simplicial $R$-module $F$, such that $F_{0}$ is free and finitely generated over $R$, and a map

$$
f^{0}: M \oplus F \longrightarrow N
$$

such that

$$
f_{*}^{0}: \pi_{0}(M \oplus F) \longrightarrow \pi_{0} N
$$

is onto. Put $M^{0}=M \oplus F$ and let $N^{0}=T\left(f^{0}\right)$. Also let $g^{0}: M^{0} \longrightarrow N^{0}$ be the front inclusion and let $p^{0}: N^{0} \longrightarrow N$ be the back projection. At this point 
we have a commutative square

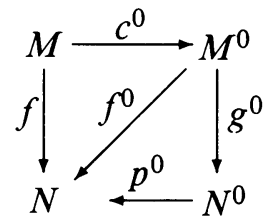

in which $c^{0}$ and $g^{0}$ are cofibrations and $p^{0}$ is a homotopy equivalence. It remains to extend the Mayer-Vietoris presentation of $M$ to a Mayer-Vietoris presentation of $M^{0}$. For this, simply note that $F_{0}$ itself may be presented in many ways as

$$
0 \longrightarrow F_{0} \stackrel{\iota}{\longrightarrow}\left(F_{A}\right)_{0} \otimes_{A} R \oplus\left(F_{B}\right)_{0} \otimes_{B} R \stackrel{\kappa}{\longrightarrow}\left(F_{C}\right)_{0} \otimes_{C} R \longrightarrow 0
$$

Extend the modules $\left(F_{A}\right)_{0},\left(F_{B}\right)_{0}$, and $\left(F_{C}\right)_{0}$ to constant simplicial modules $F_{A}, F_{B}$, and $F_{C}$, and the maps $l$ and $\kappa$ to constant simplicial maps, and put $M_{?}^{0}=M_{?} \oplus F_{?}$ for $?=A, B$, or $C$, and let $c_{?}^{0}: M_{?} \longrightarrow M_{?}^{0}$ be the inclusion of the first summand. Obviously

$$
0 \longrightarrow M^{0} \stackrel{\iota}{\longrightarrow} M_{A}^{0} \otimes_{A} R \oplus M_{B}^{0} \otimes_{B} R \stackrel{\kappa}{\longrightarrow} M_{C}^{0} \otimes_{C} R \longrightarrow 0
$$

is a Mayer-Vietoris presentation, and the quadruple $\left(c^{0}, c_{A}^{0}, c_{B}^{0}, c_{C}^{0}\right)$ is a cofibration of Mayer-Vietoris presentations

$$
\left(c^{0}, \ldots\right):\left(M, M_{A}, M_{B}, M_{C}, l, \kappa\right) \longrightarrow\left(M^{0}, M_{A}^{0}, M_{B}^{0}, M_{C}^{0}, \imath, \kappa\right) .
$$

Now let $n \geq 0$ and assume that we have constructed a diagram

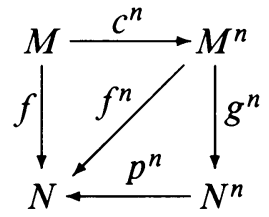

in which $f^{n}: M^{n} \longrightarrow N$ is an $n$-connected map and where $c^{n}$ is part of a cofibration of Mayer-Vietoris presentations

$$
\left(c^{n}, \ldots\right):\left(M, M_{A}, M_{B}, M_{C}, l, \kappa\right) \longrightarrow\left(M^{n}, M_{A}^{n}, M_{B}^{n}, M_{C}^{n}, l, \kappa\right) .
$$

We take $N^{n}=T\left(f^{n}\right)$ to be the mapping cylinder of $f^{n}$, and we let $g^{n}$ and $p^{n}$ be the front inclusion and back projection of the mapping cylinder, respectively. We say that $f^{n}$ is $n$-connected if the pair $\left(N^{n}, g^{n}\left(M^{n}\right)\right)$ is $n$ connected. This means, in turn, that every component of $N^{n}$ meets a component of $g^{n}\left(M^{n}\right)$ and, for any basepoint in $M^{n}$, the relative homotopy groups $\pi_{k}\left(N^{n}, g^{n}\left(M^{n}\right)\right)=0$ for $1 \leq k \leq n$. However, the homotopy groups of a simplicial abelian group relative to an arbitrary basepoint are isomorphic to those relative to the basepoint zero. Therefore, in this section and in the next, all homotopy groups are defined with respect to the zero basepoint.

To return to the argument, we have

$$
0 \longrightarrow M^{n} \stackrel{g^{n}}{\longrightarrow} N^{n} \longrightarrow N^{n} / M^{n} \longrightarrow 0,
$$

which is a simplicial fibration of $R$-modules over a connected base $N^{n} / M^{n}$. Moreover, $\pi_{k}\left(N^{n} / M^{n}\right)=0$ for $0 \leq k \leq n$. We find that the $R$-module 
$\pi_{n+1}\left(N^{n} / M^{n}\right)$ is a quotient of a finitely generated stably free $R$-module by 3.6 , so that finitely many simplicial maps

$$
\Delta^{n+1} / \partial \Delta^{n+1} \longrightarrow N^{n} / M^{n}
$$

suffice to represent a set of $R$-module generators for the group. Consider any one of these maps as a map of pairs

$$
\left(\Delta^{n+1}, \partial \Delta^{n+1}\right) \longrightarrow\left(N^{n} / M^{n}, 0\right)
$$

and let $\Lambda$ denote the horn of the simplex formed by taking all faces except $d_{0}\left(\sigma_{n+1}\right)$. By the lifting property for simplicial fibrations we may lift such an arrow to the diagonal one in the following diagram:

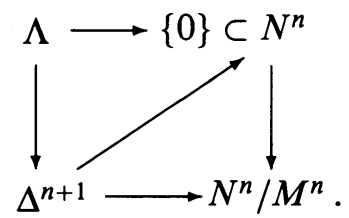

This procedure transforms a single map into a diagram of maps

$$
\begin{array}{ccc}
\left(\partial \Delta^{n+1}, \Lambda\right) & \longrightarrow & \left(M^{n}, 0\right) \\
\downarrow & & \downarrow \\
\left(\Delta^{n+1}, \Lambda\right) & \longrightarrow & \left(N^{n}, 0\right)
\end{array}
$$

Since $\pi_{n+1}\left(N^{n} / M^{n}\right)$ is finitely generated as an $R$-module, we may choose a finitely generated free module $F^{n}$ and construct a diagram of simplicial $R$ modules and homomorphisms

$$
\begin{array}{ccc}
\left(\partial \Delta^{n+1}, \Lambda\right) F^{n} & \longrightarrow & \left(M^{n}, 0\right) \\
\downarrow & & \downarrow \\
\left(\Delta^{n+1}, \Lambda\right) F^{n} & \longrightarrow & \left(N^{n}, 0\right) .
\end{array}
$$

such that the induced map on relative homotopy groups is surjective. Then the usual coning-off process displayed in the pushout diagram

$$
\begin{array}{ccc}
{\left[\partial \Delta^{n+1}\right] F^{n} /[\Lambda] F^{n}} & \stackrel{\varphi}{\longrightarrow} & M^{n} \\
\downarrow & & \downarrow \\
{\left[\Delta^{n+1}\right] F^{n} /[\Lambda] F^{n}} & \stackrel{\psi}{\longrightarrow} & M^{n+1},
\end{array}
$$

defines the $R$-module $M^{n+1}$ and a cofibration $M^{n} \longrightarrow M^{n+1}$. The universal property of the pushout yields an $(n+1)$-connected map $f^{n+1}: M^{n+1} \longrightarrow N$ which may be factored over its mapping cylinder $T\left(g^{n+1}\right)=N^{n+1}$ as before, so that we now have the commuting diagram

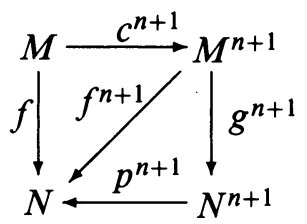

in which the right-hand vertical arrow $g^{n+1}: M^{n+1} \longrightarrow N^{n+1}$ is an $(n+1)$ connected cofibration. 
It remains to extend the Mayer-Vietoris presentation $\left(M^{n}, M_{A}^{n}, M_{B}^{n}, M_{C}^{n}\right.$, $l, \kappa)$ to a Mayer-Vietoris presentation of $M^{n+1}$. Since maps into MayerVietoris presentations may be added, it suffices to explain how this works with $F^{n}$ of rank one. In this case we have a diagram

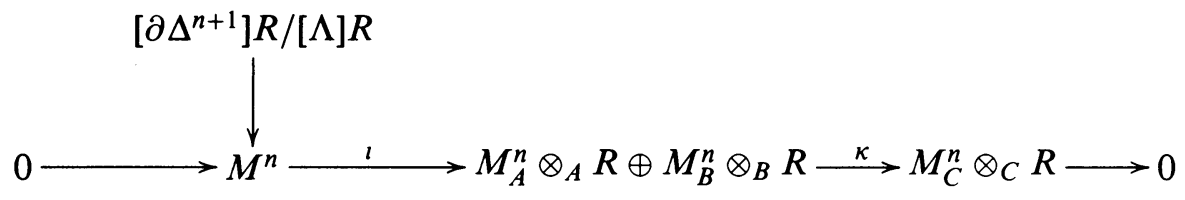

which we would like to complete to the following diagram:

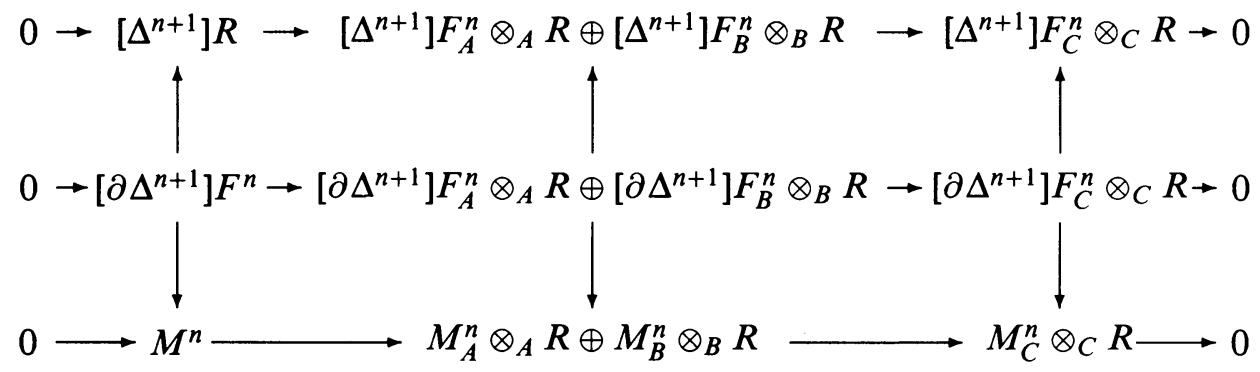

(For simplicity we have omitted the horn $[\Lambda] R$ from the diagram, as it just maps to zero.) To obtain the downward-pointing arrows we just apply Proposition 3.1 , and to obtain the upward-pointing arrows we apply the naturality of the construction [-]M in the space variable. Then the cofibration

$$
\left(c^{n+1}, \ldots\right):\left(M, M_{A}, M_{B}, M_{C}, \imath, \kappa\right) \longrightarrow\left(M^{n+1}, M_{A}^{n+1}, M_{B}^{n+1}, M_{C}^{n+1}, \imath, \kappa\right)
$$

is gotten from the pushout construction in the category $\mathscr{M} V$.

Since $M$ and $N$ are finite dimensional, inductively applying the preceding construction eventually yields a cofibration of Mayer Vietoris presentations

$$
\left(c^{d}, \ldots\right):\left(M, M_{A}, M_{B}, M_{C}, \imath, \kappa\right) \longrightarrow\left(M^{d}, M_{A}^{d}, M_{B}^{d}, M_{C}^{d}, \imath, \kappa\right)
$$

and a situation

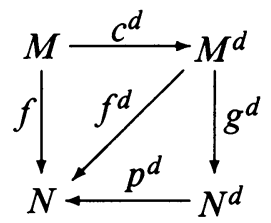

as above in which all nondegenerate simplices of $M^{d}$ and $N$ are of dimension less than or equal to $d$ and $f^{d}$ is $d$-connected. Therefore $\pi_{n}(N)=0$ for $n \geq d+1$, and the only nonvanishing homotopy group of $N^{d} / M^{d}$ is $\pi_{d+1}\left(N^{d} / M^{d}\right)$, arising as a kernel as in Lemma 3.6. From the homotopy sequence of the fibration

$$
0 \longrightarrow M^{d} \longrightarrow N^{d} \longrightarrow N^{d} / M^{d} \longrightarrow 0
$$

we extract a short exact sequence

$$
0 \longrightarrow \pi_{d+1}\left(N^{d} / M^{d}\right) \longrightarrow \pi_{d} M^{d} \longrightarrow \pi_{d} N^{d} \longrightarrow 0 .
$$


By Lemma 3.6 the $R$-module $\pi_{d+1}\left(N^{d} / M^{d}\right)$ will be stably free, so we may choose a natural number $k$ and an isomorphism

$$
R^{l} \stackrel{\cong}{\longrightarrow} \pi_{d+1}\left(N^{d} / M^{d}\right) \oplus R^{k}
$$

Construct $\bar{M}^{d}$ by taking the pushout

$$
\begin{array}{ccc}
{\left[\partial \Delta^{d}\right] R^{k}} & \longrightarrow & M^{d} \\
\downarrow & & \downarrow \\
{\left[\Delta^{d}\right] R^{k}} & \longrightarrow & \frac{M}{}^{d}
\end{array}
$$

where the upper horizontal map is zero. Extend $M^{d} \longrightarrow N^{d}$ to $\bar{M}^{d} \longrightarrow N^{d}$ by mapping the newly attached cells to zero. As before, we extend the MayerVietoris presentation of $M^{d}$ to one of $\bar{M}^{d}$ and consider the revised situation

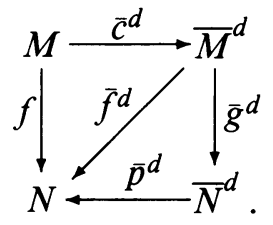

The segment of the homotopy sequence is now

$$
\begin{gathered}
R^{l} \\
\downarrow \\
\stackrel{\downarrow}{\downarrow} \pi_{d+1}\left(\bar{N}^{d} / \bar{M}^{d}\right) \longrightarrow \pi_{d} \bar{M}^{d} \longrightarrow \pi_{d} \bar{N}^{d} \longrightarrow 0
\end{gathered}
$$

in which the vertical map is an isomorphism. If we use this isomorphism to pick a minimal number of generators for $\pi_{d+1}\left(\bar{N}^{d} / \bar{M}^{d}\right)$, add $(d+1)$-cells to $\bar{M}^{d}$ accordingly, and extend the map to $N$, then a calculation using the exact homotopy sequence of the triple $\left(\bar{N}^{d}, M^{d+1}, \bar{M}^{d}\right)$ shows that we kill exactly this last kernel without introducing any homotopy in the next higher dimension, thereby producing an equivalence $M^{d+1} \longrightarrow N$. As before, we extend the Mayer-Vietoris presentation of $\bar{M}^{d}$ to one of $M^{d+1}$. At this point the improvement procedure has produced the required data, so we terminate the construction.

The proof that the forgetful functor $u_{R}$ satisfies App 2 is then complete. By application of Theorem 5 we conclude that the forgetful functor $u_{R}$ induces a homotopy equivalence

$$
w S_{\bullet} \mathscr{M} V \longrightarrow h S_{\bullet} \mathscr{M}_{f}(R),
$$

as claimed.

\section{5. $K$-THEORY OF SPLIT MODULES}

Let $\mathscr{S}$ denote the subcategory of Mayer-Vietoris presentations of the trivial $R$-module, also known as the category of split modules. One of our standard 
Mayer-Vietoris presentations is in $\mathscr{S}$ if and only if the map $\kappa$ is an isomorphism. $\mathscr{M} V^{w}$ is the subcategory of the category of Mayer-Vietoris presentations in which the $R$-module $M$ is contractible. We are going to prove the following theorem.

Theorem 3. The inclusion functor

$$
i: \mathscr{S} \longrightarrow \mathscr{M} V^{w}
$$

induces a homotopy equivalence

$$
v S_{\mathscr{O}} \mathscr{S} \longrightarrow v S_{\mathscr{O}} \mathscr{M} V^{w} .
$$

Proof. This is also an application of Theorem 5, the approximation theorem. Once again the condition $A p p 1$ is satisfied by definition of the class of weak equivalences $v$, so we have to check App 2. In this situation we are given a map $\left(0, f_{A}, f_{B}, f_{C}\right)$ of Mayer-Vietoris presentations

$$
\begin{aligned}
& 0 \longrightarrow 0 \quad \longrightarrow \quad M_{A} \otimes_{A} R \oplus M_{B} \otimes_{B} R \longrightarrow M_{C} \otimes_{C} R \longrightarrow 0 \\
& 0 \longrightarrow \stackrel{\downarrow}{N} \longrightarrow N_{A} \otimes_{A} R \stackrel{\downarrow}{\oplus} N_{B} \otimes_{B} R \longrightarrow N_{C} \otimes_{C} R \longrightarrow 0
\end{aligned}
$$

in which $N$ is contractible, and we seek a cofibration in $\mathscr{S}$,

$$
\left(0, c_{A}, c_{B}, c_{C}\right):\left(0, M_{A}, M_{B}, M_{C}\right) \longrightarrow\left(0, M_{A}^{\prime}, M_{B}^{\prime}, M_{C}^{\prime}\right)
$$

and a $v$-equivalence

$$
\left(0, f_{A}^{\prime}, f_{B}^{\prime}, f_{C}^{\prime}\right):\left(0, M_{A}^{\prime}, M_{B}^{\prime}, M_{C}^{\prime}\right) \longrightarrow\left(N, N_{A}, N_{B}, N_{C}\right)
$$

such that

$$
\left(0, f_{A}, f_{B}, f_{C}\right)=\left(0, f_{A}^{\prime}, f_{B}^{\prime}, f_{C}^{\prime}\right) \circ\left(0, c_{A}, c_{B}, c_{C}\right) .
$$

This we do stepwise, as before.

Given a finite set of $r$ classes in $\pi_{0}\left(N_{C}\right)$ which represent generators of $\pi_{0}\left(N_{C}\right)$ modulo the image of $\pi_{0}\left(M_{C}\right)$, we may represent each class by a map $\Delta^{0} \longrightarrow N_{C}$. Consider the diagram

$$
0 \longrightarrow N \longrightarrow N_{A} \otimes_{A} R \oplus N_{B} \otimes_{B} R \longrightarrow \begin{gathered}
{\left[\Delta^{0}\right] C^{r} \otimes_{C} R} \\
\downarrow \\
N_{C} \otimes_{C} R
\end{gathered} \longrightarrow 0 .
$$

Applying Proposition $3.4 r$ times in the case $n=0$, taking direct sums, and then extending the domain to a constant simplicial module, we obtain the following map:

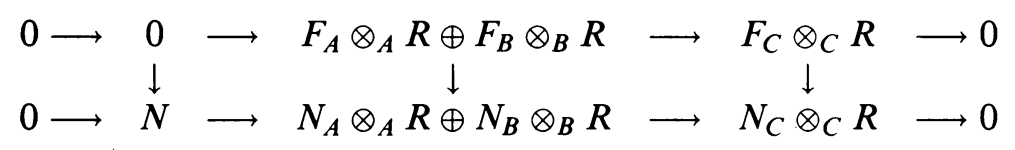

whose domain is a split module, and such that the given set of classes in $\pi_{0}\left(N_{C}\right)$ is in the image of $F_{C} \longrightarrow N_{C}$. 
If we now add this map to the original one, putting $M_{A}^{0}=M_{A} \oplus F_{A}, M_{B}^{0}=$ $M_{B} \oplus F_{B}$, and $M_{C}^{0}=M_{C} \oplus F_{C}$, then we obtain a diagram

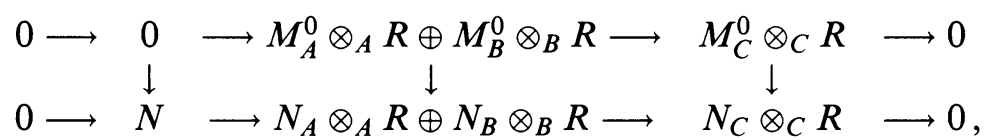

and we have extended our original map of Mayer-Vietoris presentations to one for which $\pi_{0}\left(M_{C}^{0}\right) \longrightarrow \pi_{0}\left(N_{C}\right)$ is surjective.

Consequently,

$$
\left(f_{C}^{0} \otimes_{C} R\right)_{*}: \pi_{0}\left(M_{C}^{0} \otimes_{C} R\right) \longrightarrow \pi_{0}\left(N_{C} \otimes_{C} R\right)
$$

is also surjective, and from the contractibility of $N$ it follows that the other maps $f_{A}^{0} \otimes_{A} R$ and $f_{B}^{0} \otimes_{B} R$ also induce surjections on $\pi_{0}$ Finally, since $R$ is free over $A$ and $B$, the maps

$$
f_{A}^{0}: M_{A}^{0}=M_{A} \oplus F_{A} \longrightarrow N_{A} \text { and } f_{B}^{0}: M_{B}^{0}=M_{B} \oplus F_{B} \longrightarrow N_{B}
$$

also induce surjections on $\pi_{0}$. Also, at this stage we can factor $\left(0, f_{A}^{0}, f_{B}^{0}, f_{C}^{0}\right)$ through its mapping cylinder, so that it becomes a cofibration, which is convenient for describing higher relative homotopy groups.

Note that in the preceding paragraph we made application of the freeness hypothesis on the original diagram of rings and its consequences for the ring $R$. At similar points in the rest of the argument we must again make use of the freeness hypothesis.

Let $n \geq 1$ and suppose that we have

$$
\left(0, f_{A}, f_{B}, f_{C}\right)=\left(0, f_{A}^{n-1}, f_{B}^{n-1}, f_{C}^{n-1}\right) \circ\left(0, c_{A}^{n-1}, c_{B}^{n-1}, c_{C}^{n-1}\right),
$$

a factorization of our original map $\left(0, f_{A}, f_{B}, f_{C}\right)$ through a cofibration and an $(n-1)$-connected map. Here, saying $\left(0, f_{A}^{n-1}, f_{B}^{n-1}, f_{C}^{n-1}\right)$ is $(n-1)$ connected means that the three maps $f_{A}^{n-1}, f_{B}^{n-1}$, and $f_{C}^{n-1}$ are all $(n-1)$ connected. We will also have that $\left(0, f_{A}^{n-1}, f_{B}^{n-1}, f_{C}^{n-1}\right)$ itself is the composition

$$
\left(0, f_{A}^{n-1}, f_{B}^{n-1}, f_{C}^{n-1}\right)=\left(\mathrm{Id}, p_{A}^{n-1}, p_{B}^{n-1}, p_{C}^{n-1}\right) \circ\left(0, g_{A}^{n-1}, g_{B}^{n-1}, g_{C}^{n-1}\right),
$$

where

$$
\begin{aligned}
\left(0, g_{A}^{n-1}, g_{B}^{n-1}, g_{C}^{n-1}\right):\left(0, M_{A}^{n-1}, M_{B}^{n-1},\right. & \left.M_{C}^{n-1}\right) \\
& \longrightarrow\left(N, N_{A}^{n-1}, N_{B}^{n-1}, N_{C}^{n-1}\right)
\end{aligned}
$$

is an $(n-1)$-connected cofibration and

$$
\left(\mathrm{Id}, p_{A}^{n-1}, p_{B}^{n-1}, p_{C}^{n-1}\right):\left(N, N_{A}^{n-1}, N_{B}^{n-1}, N_{C}^{n-1}\right) \longrightarrow\left(N, N_{A}, N_{B}, N_{C}\right)
$$

is a fine equivalence. This factorization $f=p \circ g$ is obtained using the mapping cylinder construction.

Now we need to improve the connectivity in this situation. Interpreting homotopy elements of the base of the fibration

$$
0 \longrightarrow M_{C}^{n-1} \longrightarrow N_{C}^{n-1} \longrightarrow N_{C}^{n-1} / M_{C}^{n-1} \longrightarrow 0
$$


as in the proof of Theorem 2, we find again that there are a finitely generated free $C$-module $L_{C}$ and a diagram of simplicial maps

$$
\begin{array}{ccc}
{\left[\partial \Delta^{n}, \Lambda\right] L_{C}} & \longrightarrow & M_{C}^{n-1} \\
\downarrow & & \downarrow \\
{\left[\Delta^{n}, \Lambda\right] L_{C}} & \longrightarrow & N_{C}^{n-1},
\end{array}
$$

where $\Lambda$ maps to zero, which one may use to improve the connectivity of $f_{C}^{n-1}$ by adding cells to $M_{C}^{n-1}$. But we are required to add cells to the whole MayerVietoris presentation $\left(0, M_{A}^{n-1}, M_{B}^{n-1}, M_{C}^{n-1}\right)$, so the argument continues as follows.

For simplicity of notation fix attention on the submodule of $\pi_{n}\left(N_{C}^{n-1}, M_{C}^{n-1}\right)$ generated by a single element, and fix attention on a single component pointed by $m$, so that the starting situation simplifies to a diagram

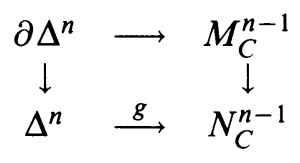

where the horn $\Lambda$ is mapped to zero. Apply Proposition 3.4 to change $g$ by a homotopy relative to $\partial \Delta^{n}$ and to complete the resulting diagram to a diagram of maps of Mayer-Vietoris presentations

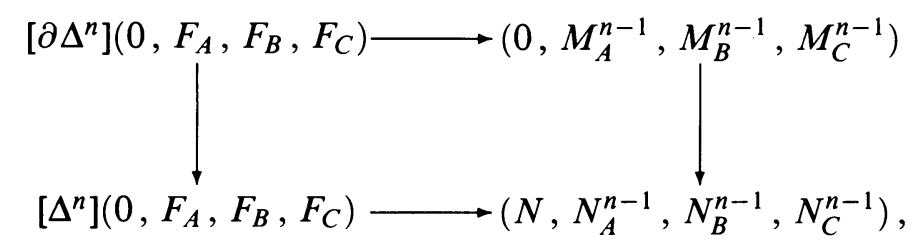

where $[\Lambda]\left(0, F_{A}, F_{B}, F_{C}\right)$ is mapped trivially. Take the pushout of the lefthand cofibration along the upper arrow in the category $\mathscr{S}$ and denote it provisionally by $\left(0, M_{A}^{n}, M_{B}^{n}, M_{C}^{n}\right)$. Continuing provisional notations, let $\left(N, N_{A}^{n}\right.$, $N_{B}^{n}, N_{C}^{n}$ ) be the mapping cylinder of

$$
\left(0, M_{A}^{n}, M_{B}^{n}, M_{C}^{n}\right) \longrightarrow\left(N, N_{A}^{n-1}, N_{B}^{n-1}, N_{C}^{n-1}\right) .
$$

Observe that the relative homotopy group $\pi_{n}\left(N_{C}^{n}, M_{C}^{n}\right)$ is a quotient of the old group $\pi_{n}\left(N_{C}^{n-1}, M_{C}^{n-1}\right)$ by a $C$-submodule containing that submodule generated by our chosen element. Since $\pi_{n}\left(N_{C}^{n-1}, M_{C}^{n-1}\right)$ is finitely generated, after finitely many applications of the procedure we have extended $\left(0, M_{A}^{n-1}, M_{B}^{n-1}\right.$, $\left.M_{C}^{n-1}\right)$ to a split module $\left(0, M_{A}^{n}, M_{B}^{n}, M_{C}^{n}\right)$ such that the relative homotopy group $\pi_{n}\left(N_{C}^{n}, M_{C}^{n}\right)$ is zero. Consequently,

$$
\begin{aligned}
0 & =\pi_{n}\left(N_{C}^{n} \otimes_{C} R, M_{C}^{n} \otimes_{C} R\right) \\
& \cong \pi_{n}\left(N_{A}^{n} \otimes_{A} R, M_{A}^{n} \otimes_{A} R\right) \oplus \pi_{n}\left(N_{B}^{n} \otimes_{B} R, M_{B}^{n} \otimes_{B} R\right)
\end{aligned}
$$

and we use the freeness of $R$ over $B$ and $A$ to conclude that $\pi_{n}\left(N_{A}^{n}, M_{A}^{n}\right)$ and $\pi_{n}\left(N_{B}^{n}, M_{B}^{n}\right)$ are also now both zero. This ends the explanation of the inductive step of the improvement procedure.

The finiteness hypothesis on all the modules, together with Lemma 3.6, brings the procedure to a halt. Applying the inductive step and using the finiteness 
hypothesis, we may assume that the only nonvanishing relative homotopy groups are in dimension $d+1$ and that all of the cells involved in building up the modules $M_{A}^{d}, M_{B}^{d}, M_{C}^{d}, N_{A}^{d}, N_{B}^{d}$, and $N_{C}^{d}$ are of dimension less than or equal to $d$. So we have a relative homotopy group

$$
Z_{C}=\pi_{d+1}\left(N_{C}^{d}, M_{C}^{d}\right) \cong \operatorname{Kernel}\left(\pi_{d}\left(M_{C}^{d}\right) \longrightarrow \pi_{d}\left(N_{C}^{d}\right)\right),
$$

and similarly defined $A$ - and $B$-modules $Z_{A}$ and $Z_{B}$. By Lemma 3.6 each of these modules is stably free and finitely generated, so we may attach $d$-cells to $\left(0, M_{A}^{d}, M_{B}^{d}, M_{C}^{d}\right)$ by trivial maps and extend the map to $\left(N, N_{A}^{d}, N_{B}^{d}, N_{C}^{d}\right)$ by making it zero on the new cells to make first $Z_{C}$ and then $Z_{A}$ and $Z_{B}$ free while they remain finitely generated. Assume that this has been done.

The prodedure for killing these last relative homotopy groups is different from that used in the inductive step, for we are now dealing with kernels. First we have a diagram

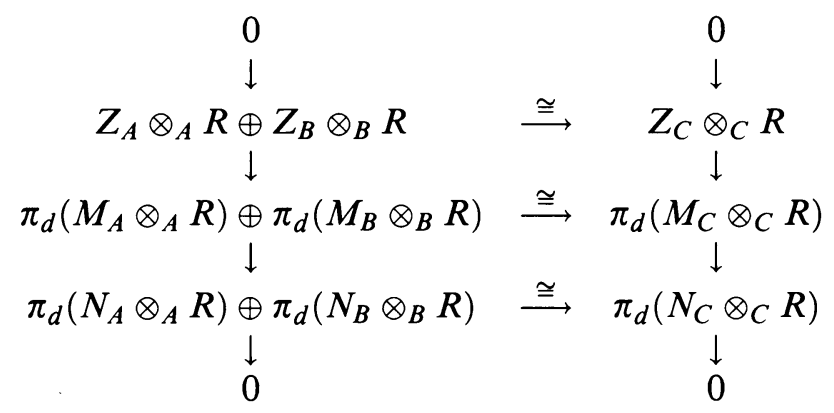

with exact columns, by exactness of all tensor products with $R$. For any choice of $d$-simplices $z_{1}, \ldots, z_{r}$ of $M_{C}$ satisfying $d_{i} z_{k}=0$ for all $i$ and $k$ and such that the homotopy classes $\left[z_{1}\right], \ldots,\left[z_{r}\right]$ provide a $C$-basis for $Z_{C}$, we get a map of split modules

$$
\left[\partial \Delta^{d+1} / \Lambda\right]\left(0, Z_{A}, Z_{B}, Z_{C}\right) \longrightarrow\left(0, M_{A}, M_{B}, M_{C}\right) .
$$

Besides using the fact that under our freeness hypotheses on $A, B$, and $C$ a map of split modules has a kernel which is also a split module, we need to know $Z_{A}, Z_{B}$, and $Z_{C}$ are finitely generated, so that the kernel is indeed in $\mathscr{S}$, but this has been guaranteed by Lemma 3.6. By the lifting argument found at the beginning of the proof of Proposition 3.4, there is a choice $z_{1}^{\prime}, \ldots, z_{r}^{\prime}$ such that the map just described extends to a diagram

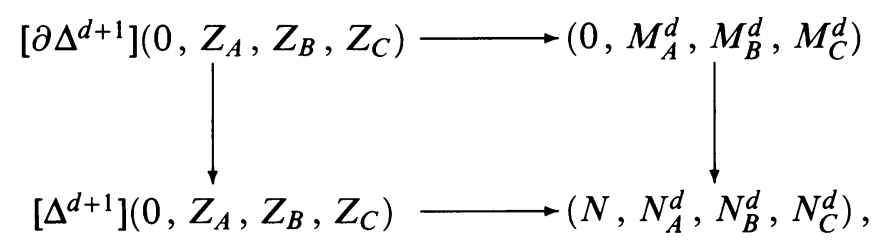

in which the left-hand vertical arrow is obviously a cofibration. Now take the pushout of this cofibration along the upper horizontal arrow and denote it by $\left(0, M_{A}^{d+1}, M_{B}^{d+1}, M_{C}^{d+1}\right)$. We also define $\left(N, N_{A}^{d+1}, N_{B}^{d+1}, N_{C}^{d+1}\right)$ to be the mapping cylinder of the canonical map of the pushout to the lower right corner. 
Clearly $\pi_{*}\left(N_{C}^{d+1}, M_{C}^{d+1}\right)=0$, so the usual argument provides equalities

$$
\pi_{*}\left(N_{A}^{d+1}, M_{A}^{d+1}\right)=0 \text { and } \pi_{*}\left(N_{B}^{d+1}, M_{B}^{d+1}\right)=0 .
$$

We have therefore completed the verification of $A p p 2$ for the inclusion $\mathscr{S} \longrightarrow$ $\mathscr{M} V^{w}$. Then, by Theorem 5

$$
v S_{\bullet} \mathscr{S} \longrightarrow v S_{\bullet} \mathscr{M} V^{w}
$$

is a homotopy equivalence, as desired.

We conclude by remarking that the obstruction to making everything in this paper work directly for particular diagrams of simplicial rings is found here in this section at those points where we want to deduce that a map of $A$-modules is highly connected, say, given that the map tensored up to $R$ is highly connected. Appropriate modifications of the arguments are discussed in [5].

\section{REFERENCES}

1. Randy McCarthy, On fundamental theorems of algebraic K-theory, Topology 32 (1993), 325-328.

2. John Milnor, Whitehead torsion, Bull. Amer. Math. Soc. 72 (1966), 358-426.

3. D.G. Quillen, Homotopical algebra, Lecture Notes in Math., vol. 43, Springer-Verlag, Berlin and New York, 1967.

4. Roland Schwänzl, Ross E. Staffeldt, and Friedhelm Waldhausen, Stable algebraic K-theory and topological Hochschild homology of $A_{\infty}$-rings, Preprint 94-003, SFB 343, Bielefeld.

5 . Decomposition theorems for $K$-theory of simplicial rings, in preparation.

6. R.E. Staffeldt, On fundamental theorems of algebraic K-theory, K-Theory 1 (1989), 511532.

7. Pierre Vogel, On the vanishing of Nil-groups, unpublished manuscript, 1990.

8. F. Waldhausen, Algebraic K-theory of generalized free products. I, II, Ann. of Math. 108 (1978), 135-256.

9. Algebraic K-theory of spaces, Lecture Notes in Math., vol. 1126, Springer-Verlag, Berlin and New York, 1983, pp. 318-419.

FAChbereich Mathematik, Universität OsNabrück, 49069 OsNABrüCK, Germany E-mail address: rolandQscarlett.mathematik.uni-osnabrueck.de

Department of Mathematical Sciences, New Mexico State University, Las Cruces, New MEXICO 88003

E-mail address: ross@nmsu.edu 